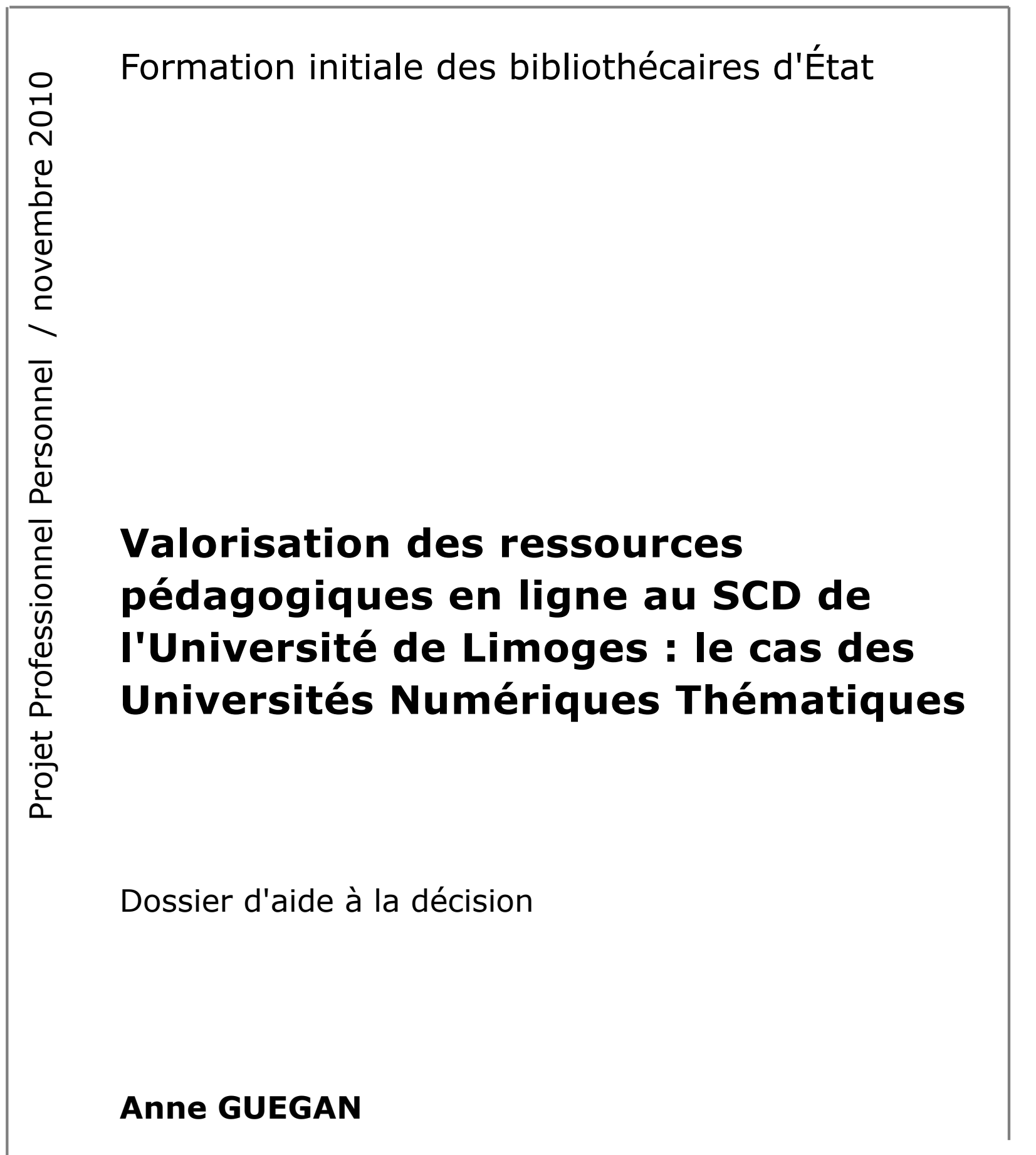

\title{
Anne GUEGAN
}

Sous la direction de Thomas Chaimbault, Bibliothèque et document numérique - ENSSIB Correspondant Établissement : Claudie Madjarev, Directrice par interim du SCD de Limoges 



\section{Remerciements}

Merci à toute l'équipe de direction du SCD de Limoges et à tous les membres du personnel pour leur accueil chaleureux.

Je tiens à remercier tout particulièrement Claudie Madjarev qui a eu l'idée de ce sujet et me l'a proposé. Je remercie aussi Serge Paronneau de l'Université de Limoges pour avoir répondu à mes questions.

Je remercie également mon tuteur, Thomas Chaimbault pour son écoute attentive, ses conseils et sa disponibilité.

Merci également à Catherine Weill du SCD Paris-Sud Orsay et Jennifer Wolfarth de l'Université du Maine pour avoir partagé leur expérience.

Enfin, je remercie l'ENSSIB et plus particulièrement Catherine Joubert, de m'avoir autorisée à aménager mon parcours de formation afin de participer au stage de formation continue sur l'indexation des ressources pédagogiques et d'assister au colloque CIUEN 2010. Ces manifestations ont été très utiles dans ma réflexion. 


\section{Résumé :}

L'indexation et la visibilité des ressources pédagogiques en ligne constituent un enjeu pour la valorisation du patrimoine pédagogique des établissements d'enseignement supérieur. Les Universités Numériques Thématiques offrent un cadre aux projets des universités en soutenant la production de ressources mutualisées. Les Services Communs de la Documentation doivent s'inscrire dans ces politiques de publication et valorisation du patrimoine numérique grâce à leur savoir-faire en matière de référencement, indexation, archivage, accès. Ce dossier vise à proposer trois scénarios pour une aide à la décision au SCD de Limoges.

Descripteurs :

Ressources pédagogiques numériques

Universités numériques thématiques

Indexation

\section{Abstract :}

The visibility and indexing of online learning resources play an important role in promoting the educational content of higher educational institutions. The digital academic networks within the framework of the French UNTS provide the necessary environment for University projects by promoting the creation of shared resources. Using their knowledge in listing, indexing, archiving and accessing resources, University Libraries need to be in line with these publishing and promotion policies. This document will aim at presenting three solutions to help and facilitate the choices of the University Library of Limoges.

Keywords :

Online Learning ressources

Digital academic networks

Indexing 
Droits d'auteurs

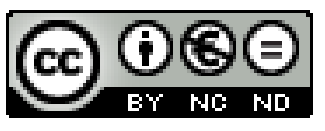

Cette création est mise à disposition selon le Contrat :

Paternité-Pas d'Utilisation Commerciale-Pas de Modification 2.0 France

disponible en ligne $h t t p: / / c r e a t i v e c o m m o n s . o r g / l i c e n s e s / b y-n c-n d / 2.0 / f r /$ ou par courrier postal à Creative Commons, 171 Second Street, Suite 300, San Francisco, California 94105, USA. 



\section{Table des matières}

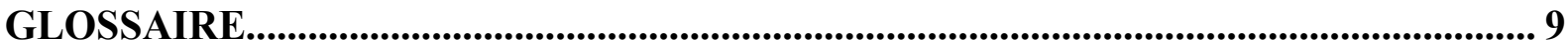

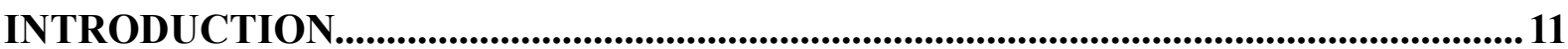

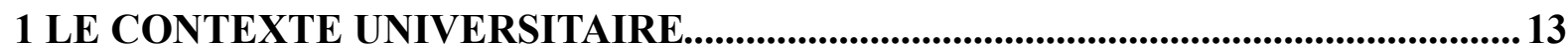

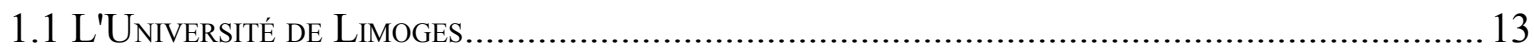

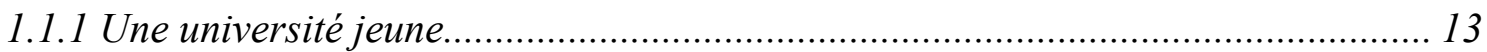

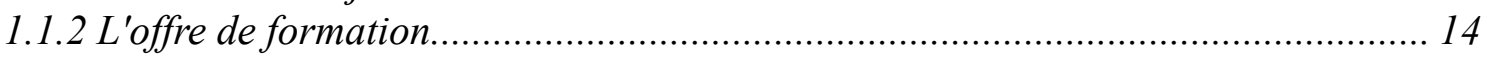

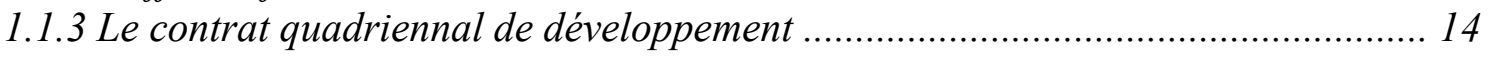

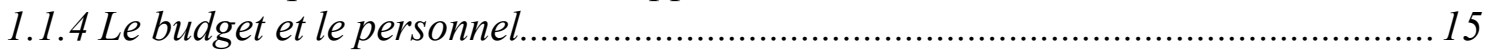

1.1.5 La recherche concentrée sur des domaines d'excellence..................................... 15

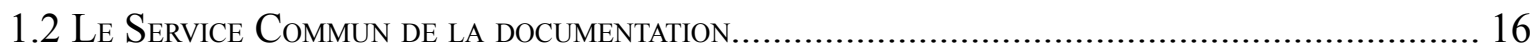

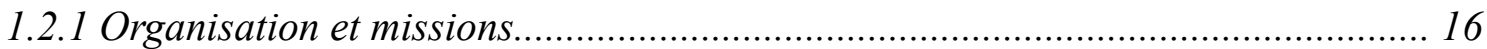

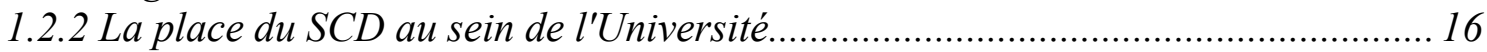

1.3 Le SCD de Limoges ET SA POLITIQUe DE VALORISATION DES RESSOURCES DE L'UNIVERSitÉ...........17

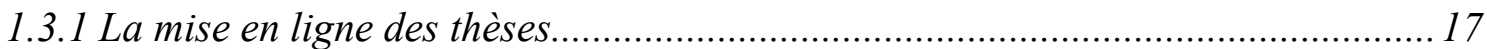

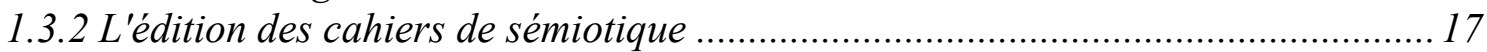

1.3.3 La numérisation des herbiers........................................................................... 17

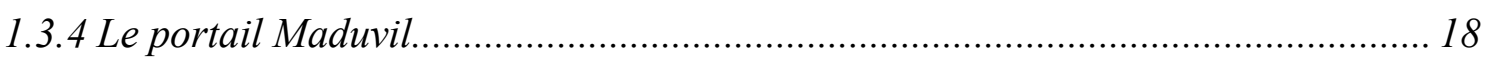

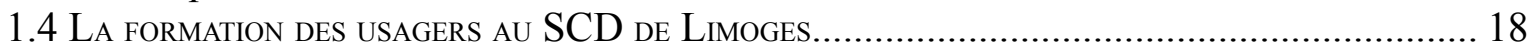

2 LES RESSOURCES PÉDAGOGIQUES NUMÉRIQUES...........................................19

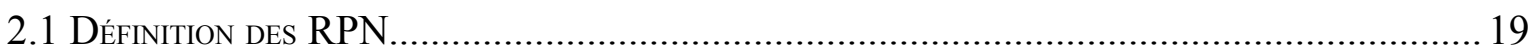

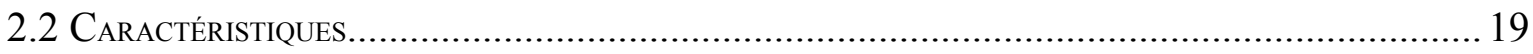

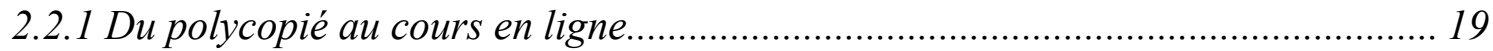

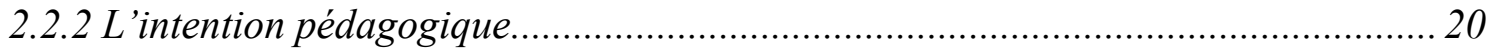

2.2.3 La granularité

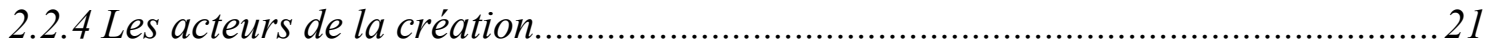

2.2.5 Le coût des ressources pédagogiques................................................................... 21

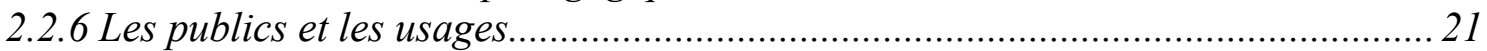

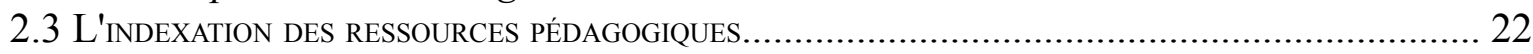

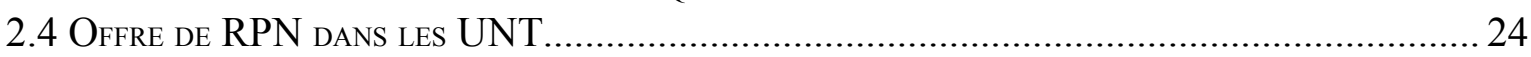

2.4.1 La mise en place des UNT ............................................................................... 24

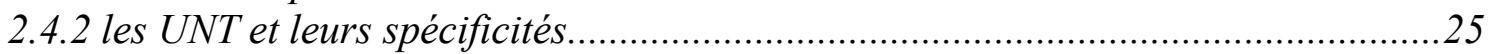

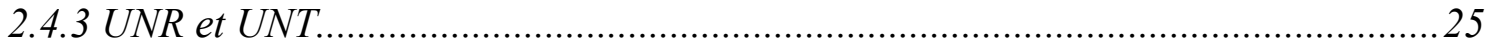

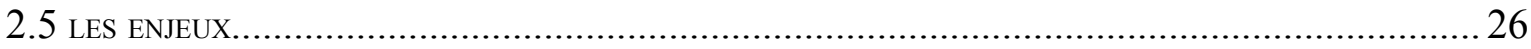

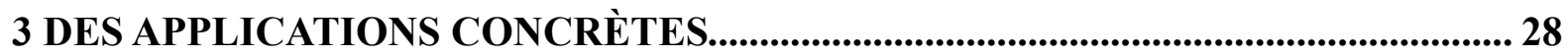

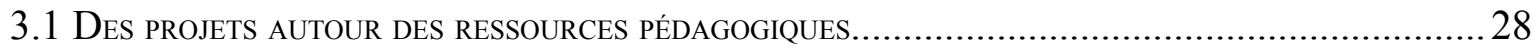

3.1.1 Le projet indexation des ressources pédagogiques au SCD Paris-Sud 11, Orsay..28

3.1.2 Université du Maine : un projet porté par l'UNR ............................................... 30

3.1.3 IUT en ligne : augmenter les usages par le cartable numérique..............................31

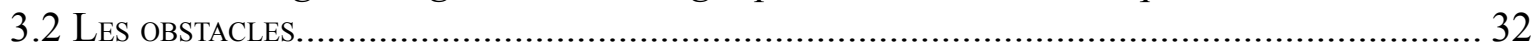

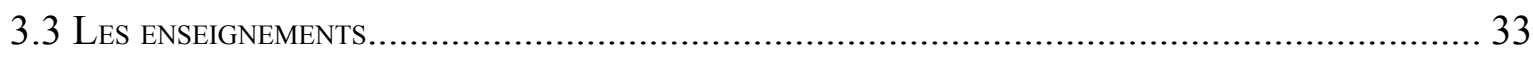

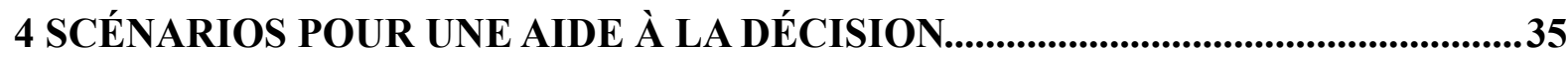

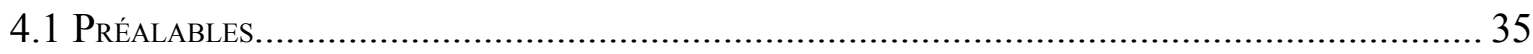




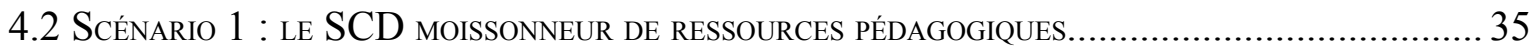

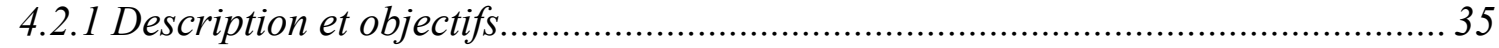

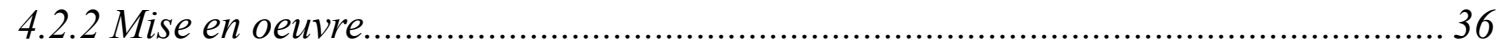

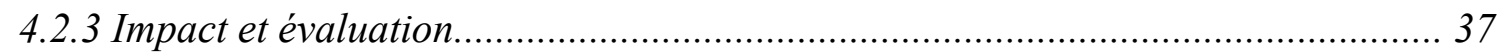

4.3 SCÉNARIo 2 : LE SCD RÉFÉRENCEUR ET INDEXEUR DE RESSOURCES PÉDAGOGIQUES.......................... 37

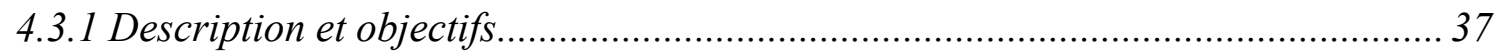

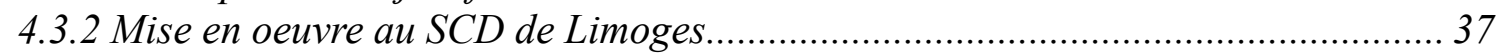

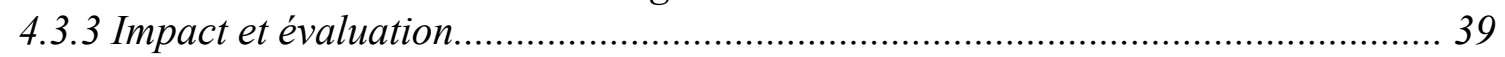

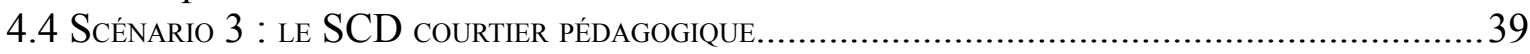

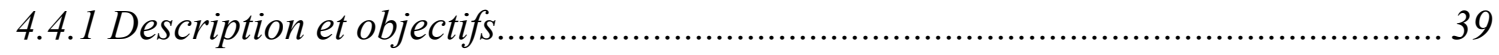

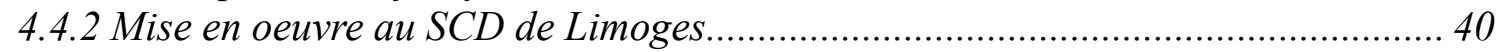

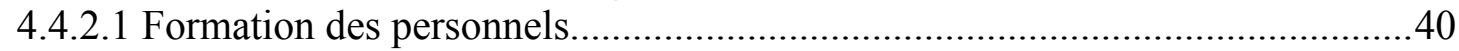

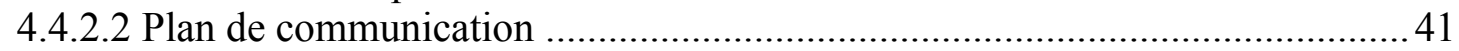

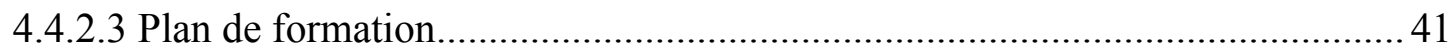

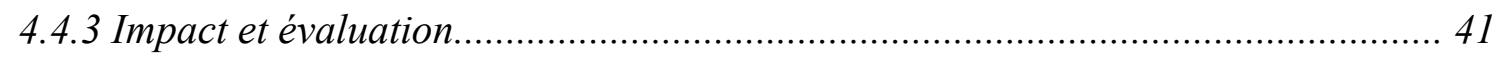

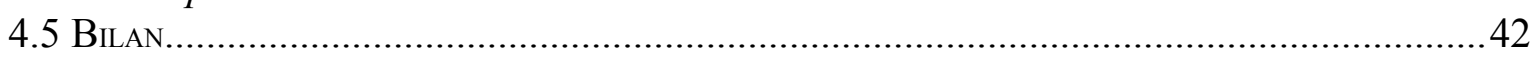

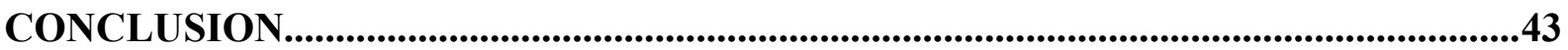

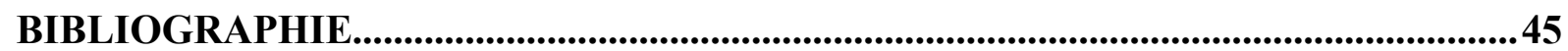

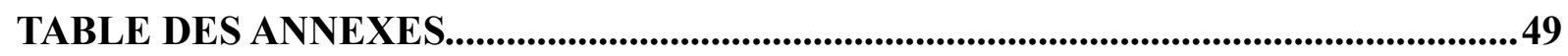


AUNEGE : Association des Universités pour l'enseignement Numérique en Economie et GEstion.

FOAD : Formation Ouverte et à Distance

GED : Gestion électronique des documents

LMS : Learning Management System

LOM : Learning Object Metadata

LOMFR : Profil français d'application du Learning Object Metadata

OAI : Open Archives Initiative

OAI-PMH : Open Archives Initiative - Protocol for Metadata Harvesting

ORI-OAI : Outil de référencement et d'indexation pour un réseau de portail OAI

RPN : Ressources pédagogiques numériques

SupLOMFR : Adaptation du LOMFR à l'enseignement supérieur

TICE : Technologies de l'Information et de la Communication pour l'Enseignement

UMVF : Université Médicale Virtuelle Francophone

UNF3S : Université Numérique Francophone des Sciences de la Santé et du Sport

UNISCIEL : Université des SCIences En Ligne

UNIT : Université Numérique Ingénierie et Technologie

UNJF : Université numérique juridique francophone

UNR : Université Numérique Régionale

UNT : Université Numérique Thématique

UOH : Université Ouverte des Humanités

UVED : Université Virtuelle Environnement et Développement Durable

XML : Extensible Markup Language 



\section{Introduction}

En octobre 2008, Valérie Pécresse, ministre de l'Enseignement supérieur et de la Recherche, s'est exprimée devant les présidents d'université, à l'occasion de l'introduction de la conférence-débat "Université numérique "1. Elle a insisté sur la nécessité d'intégrer le numérique dans la politique des universités au service de la pédagogie. A cette fin, elle incite très fortement les universités à créer un "véritable patrimoine de la documentation numérique " avec des cours enregistrés, des documents numérisés ou encore des " serious games " et à se doter des outils nécessaires pour les rendre visibles et accessibles.

Il est acquis depuis une décennie que l'offre de documentation des Services Communs de Documentation comprend des ressources numériques : revues, thèses, bases de données, ebooks. A cet effet, les $\mathrm{SCD}^{2}$ se sont dotés d'outils pour rendre visibles ces ressources numériques : métamoteur, moissonneur $\mathrm{OAI}^{3}$, résolveur de liens ou $\mathrm{GED}^{4}$. Or, les ressources pédagogiques numériques trouvent plus rarement leur place dans l'offre des SCD. Consciente des enjeux liés à l'accessibilité de ce type de ressources, la direction du SCD de $\operatorname{Limoges}^{5}$ a évoqué cette question à plusieurs reprises sans que des solutions ne soient mises en place.

C'est pour cette raison que Claudie Madjarev, Conservateur responsable de la section DroitSciences économiques, puis Directrice par interim du SCD de Limoges, établissement où je suis affectée, m'a proposé comme sujet de projet professionnel personnel (PPP) : " La valorisation des ressources pédagogiques numériques au SCD de Limoges ". Ces ressources sont en plein développement notamment grâce aux universités numériques thématiques (UNT).

Une présentation de l'Université de Limoges avec ses particularités géographiques et humaines est nécessaire pour mieux cerner le contexte dans lequel se place ce projet. L'analyse des missions du SCD au sein de l'Université démontrera comment et avec quelles compétences le SCD contribue à valoriser les ressources de l'Université.

La deuxième partie de ce rapport traitera de la nature des ressources pédagogiques avec une attention particulière aux UNT. Il convient de définir exactement ce que l'on entend par ressources pédagogiques, leurs caractéristiques, les publics et les usages. Puis on s'interrogera sur les UNT. Quelles sont leurs spécificités et leurs liens avec les universités?

Des projets de valorisation de ressources pédagogiques ont été menés ou sont en cours dans des établissements. Ceux-ci ont mis en place des solutions qui seront examinées et

\footnotetext{
${ }^{1}$ MINISTERE DE L'ENSEIGNEMENT SUPERIEUR ET DE LA RECHERCHE. Faire du numérique un outil pédagogique et au service de la vie étudiante [en ligne]. Disponible sur : < http://www.enseignementsup-recherche.gouv.fr/cid22726/faire-du-numerique-un-outilpedagogique-et-au-service-de-la-vie-etudiante.html > (consulté le 14 Juin 2010)

${ }^{2}$ Service Commun de Documentation

${ }^{3}$ Open Archives Initiative

${ }^{4}$ Gestion Électronique des documents

${ }^{5} \mathrm{La}$ question a été posée à deux reprises : en 2008 lors d'une présentation au SCD de la plate-forme IUT en ligne, puis en 2009 la question de la valorisation des ressources de l'UNJF a été posée par Claudie Madjarev en Commission de bibliothèque. Les comptes rendus de ces réunions sont consultables sur l'intranet du SCD.
} 
commentées dans une troisième partie. Les obstacles rencontrés et les réussites sont des enseignements pour des projets à venir.

Enfin, en quatrième partie de ce rapport, trois scénarios d'aide à la décision seront proposés pour répondre à la question initiale : " La valorisation des ressources pédagogiques en ligne : le cas des UNT". 


\section{Le contexte universitaire}

\subsection{L'Université de Limoges}

Située dans le Centre-Ouest de la France, Limoges est préfecture du département de la HauteVienne et capitale de la région Limousin. Elle est internationalement renommée du fait de l'implantation historique de l'industrie de la porcelaine et de l'artisanat de l'émail et des vitraux. La ville de Limoges compte près de 140000 habitants et se situe dans une aire urbaine de 260000 habitants qui la place au 3e rang des agglomérations du grand sud-ouest après Toulouse et Bordeaux.

Unique opérateur dans sa région, l'Université de Limoges est implantée sur neuf sites dans la ville de Limoges et huit sites dans la région Limousin (trois sites en Creuse et cinq en Corrèze). L'identification de l'Université à son territoire géographique constitue l'une des forces de l'Université.

\subsubsection{Une université jeune}

L'Université de Limoges se présente elle-même comme une université jeune puisqu'elle date de 1968. Mais l'origine de l'enseignement supérieur à Limoges remonte à 1626 avec la création de la " Maîtrise des Apothicaires " et du " Collège de Médecine et de Pharmacie ". La Faculté Libre de Droit voit sa création plus tard en 1927, elle est alors liée par convention à la Faculté de Droit de Poitiers ${ }^{6}$.

Les statuts de l'Université ont été élaborés en 1970 par l'Assemblée Constitutive présidée par Monsieur Savy, professeur à la Faculté de Droit et Sciences Economiques.

En 1970, l'Université compte alors 7000 étudiants. Ses effectifs ont doublé en 40 ans puisqu'elle accueille aujourd'hui environ 14000 étudiants. Cette progression n'est pas linéaire et les effectifs montrent une tendance à la baisse depuis 2004. Artificiellement maintenus par l'apport des effectifs de l'IUFM ${ }^{7}$ intégré en 2007 et la part des étudiants étrangers en légère hausse, cette stagnation des effectifs pose la question de l'attractivité de l'Université dans et hors de sa région. La proportion d'étudiants étrangers reste inférieure à la moyenne nationale et $60 \%$ des étudiants de l'Université de Limoges ont obtenu leur baccalauréat dans l'Académie. L'Université de Limoges mentionne d'ailleurs la " concurrence" des grandes universités limitrophes ${ }^{8}$.

${ }^{6}$ UNIVERSITE DE LIMOGES. L'université de Limoges ... un peu d'Histoire [en ligne]. Disponible sur : < http://www.unilim.fr/spip.php? article658\&lang $=\mathrm{fr}>($ Consulté le 14 juin 2010)

${ }^{7}$ Institut Universitaire de Formation des Maîtres

${ }^{8}$ UNIVERSITE DE LIMOGES. Tableau de bord de l'Université : TABUL [en ligne]. Disponible sur : <http://www.unilim.fr/spip.php? article642\&lang=fr $>($ Consulté le 14 juin 2010) 


\subsubsection{L'offre de formation}

L'Université de Limoges a accueilli 14000 étudiants à la rentrée 2009 dont $60 \%$ sont originaires de la région Limousin.

L'Université de Limoges est un établissement pluridisciplinaire avec santé : un étudiant qui veut étudier dans le Limousin a le choix entre 5 UFR : droit et sciences économiques, médecine, pharmacie, lettres et sciences humaines, sciences et techniques, 4 Instituts : IUT, IAE, IPAG, IUFM (récemment intégré), 2 Ecoles d'ingénieurs, l'une intégrée et l'autre associée (l'Ecole nationale supérieure d'ingénieurs de Limoges - ENSIL, et l'Ecole nationale supérieure de céramique industrielle - ENSCI - associée).

La pluridisciplinarité de l'université permet donc théoriquement à un étudiant de rester faire ses études dans sa région, même si le rapport de l'AERES ${ }^{9}$ signale l'attractivité de plusieurs grandes universités limitrophes.

D'autre part, le relatif éloignement entre les sites limousins amène l'université à s'interroger sur les disparités dans les services offerts aux étudiants.

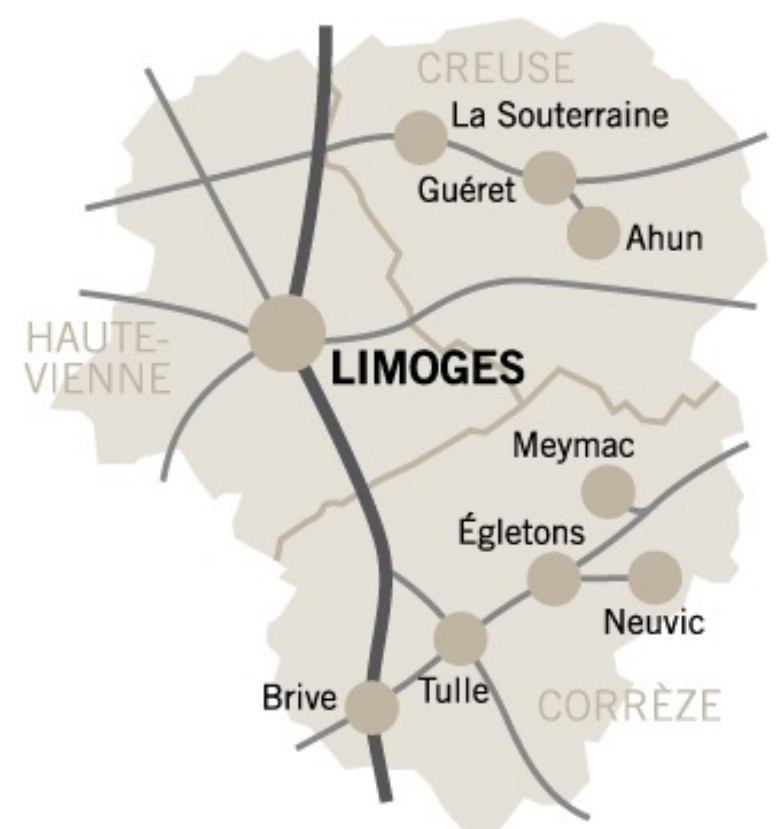

Les sites de l'Université de Limoges ${ }^{10}$

\subsubsection{Le contrat quadriennal de développement}

Le contrat quadriennal pour la période $2008-2011^{11}$ fixe quatre axes de développement dont deux sont directement liés à la problématique de cette étude. L'axe 2 fixe comme objectif de " mener les étudiants à la réussite dans leurs études et dans leur insertion professionnelle " et l'axe 3 comprend le point suivant " mobiliser les TIC au service du développement de l'établissement ".

${ }^{9}$ Agence d'évaluation de la recherche et de l'enseignement supérieur

${ }^{10}$ UNIVERSITE DE LIMOGES. Présentation. Localisation géographique [en ligne]. Disponible sur : <http://www.unilim.fr/spip.php? rubrique52\&lang=fr>. (Consulté le 24 août 2010)

${ }^{11}$ UNIVERSITE DE LIMOGES. Contrat quadriennal 2008-2011. Disponible sur l'intranet de l'Université. (Consulté le 24 août 2010 ) 
Dans cet axe 3 concernant les TIC-TICE, l'Université affirme la volonté suivante :

" En interne, les priorités de l'Université sont :

- d'assurer l'attractivité de l'Université : enrichir l'offre de formation par de la production pédagogique numérique, valoriser l'activité recherche par la mise ligne de la production scientifique (thèses, publications, édition électronique...), améliorer de façon constante le portail web institutionnel

- de continuer à orienter les TIC vers une politique de services et d'usage en :

- développant l'accompagnement, la sensibilisation et la formation des acteurs universitaires aux outils numériques dans les différents secteurs d'activité ;

- facilitant l'accès aux ressources numériques de façon permanente et en tous lieux. "

\subsubsection{Le budget et le personnel}

Les crédits ouverts au budget 2010 prenant en compte le transfert de la masse salariale globale est à hauteur de 150 millions d'euros pour l'Université de Limoges.

Les ressources sont d'origine ministérielle à $75 \%$, des collectivités territoriales et des organismes de recherche pour $10,5 \%$, de l'activité propre de l'université pour $11,5 \%$, et de provenances diverses pour 3\%. Les dépenses de personnels représentent $70 \%$ du budget, les missions de formation et de recherche $19 \%$, la mise en œuvre du pilotage : $3 \%$, les dépenses d'infrastructures : $8 \%{ }^{12}$

L'Université de Limoges compte 1463 personnels répartis en 925 enseignants et enseignantschercheurs et 538 personnels BIATOSS (personnel de bibliothèque, ingénieurs, administratifs, techniciens, ouvriers, personnels de service et de santé). ${ }^{13}$

\subsubsection{La recherche concentrée sur des domaines d'excellence}

L'Université de Limoges est aussi un important centre de recherche qui accueille 700 chercheurs autour de spécialités internationalement reconnues comme la génomique, la sémiologie, la domotique, la cryptologie, les matériaux, l'environnement et l'eau, l'électronique optique et les territoires. 


\subsection{Le Service Commun de la documentation}

\subsubsection{Organisation et missions}

Le SCD de Limoges est organisé en six sections documentaires. A ces sections, il faut ajouter des bibliothèques intégrées ou associées qui portent à douze le nombre de sites desservis par le SCD.

Les sites étant dispersés géographiquement sur l'ensemble de la région Limousin, le SCD de Limoges doit répondre à des besoins importants en personnels et en collection.

Le Service Commun de la Documentation a pour fonction de :

- $\quad$ mettre en œuvre la politique documentaire de l'Université, coordonner les moyens et évaluer les services offerts aux usagers

- $\quad$ acquérir, gérer et communiquer les documents de toutes sortes qui appartiennent à l'Université ou qui sont à sa disposition

- $\quad$ participer, à l'intention des utilisateurs, à la recherche sur ces documents, à la production de l'information scientifique et technique, à sa diffusion, ainsi qu'aux activités d'animation

- $\quad$ former les utilisateurs à un emploi aussi large que possible des techniques nouvelles d'accès à l'information scientifique et technique et coopérer avec les bibliothèques qui concourent aux mêmes objectifs, notamment par la participation à des catalogues collectifs

Ce service assure également les missions d'orientation, d'étude, de recherche et d'enseignement bibliographique et documentaire à destination des usagers. ${ }^{14}$

\subsubsection{La place du SCD au sein de I'Université}

L'insertion du SCD au sein de l'Université se concrétise par l'existence d'un conseil spécifique, le Conseil de la Documentation qui réunit des représentants de la communauté universitaire, des enseignants-chercheurs, des étudiants et des représentants du personnel du SCD. Ce conseil est un lieu de débat et d'échange, il examine le budget, se prononce sur les règles de fonctionnement et sur la politique documentaire du SCD. Le Conseil de la Documentation se réunit une à deux fois par $\mathrm{an}^{15}$.

\footnotetext{
${ }^{14}$ Source : intranet du SCD

${ }^{15}$ Conseil de la Documentation de l'Université de Limoges : comptes rendus en ligne sur l'intranet du SCD
} 


\subsection{Le SCD de Limoges et sa politique de valorisation DES RESSOURCES DE L'UNIVERSITÉ}

L'une des façons de palier le problème de la dispersion des sites est de favoriser la numérisation des collections. Cela permet de rétablir une égalité d'accès aux ressources quelle que soit la localisation géographique de l'utilisateur. En outre, la mise en ligne des collections numérisées donne de la visibilité à l'extérieur de l'Université, met l'accent sur les spécificités des collections régionales et casse le relatif isolement de la région par rapport à ses concurrentes.

\subsubsection{La mise en ligne des thèses}

Dès 2003, le SCD de Limoges a été précurseur et site pilote pour la mise en ligne des thèses électroniques. En effet, le dépôt des thèses sous forme électronique a été rendu obligatoire pour les doctorants de l'Université de Limoges ${ }^{16}$. L'usage d'une feuille de style spécifique est par ailleurs imposé afin d'assurer la structuration du document et de permettre la conversion des thèses en fichiers HTML pour la diffusion et XML pour l'archivage.

En collaboration avec les écoles doctorales, le SCD propose des formations aux doctorants : utilisation de la feuille de style, sensibilisation au droit d'auteur, traitement de texte et d'images.

Le texte intégral de l'ensemble des thèses mises en ligne est disponible sur le site du SCD de l'Université de Limoges, dans la rubrique "Thèses en ligne" du menu e-ressources. ${ }^{17}$

\subsubsection{L'édition des cahiers de sémiotique}

Les Presses universitaires de Limoges (PULIM) ${ }^{18}$ et le SCD de l'université de Limoges se sont associés pour éditer en ligne des revues scientifiques et actes de colloques.

Grâce à l'utilisation de la plate-forme Lodel et à la collaboration du Centre de Recherches Sémiotiques (CERES), une première revue a été mise en ligne en mai 2007 : les Nouveaux Actes Sémiotiques. ${ }^{19}$ Le site des Nouveaux Actes Sémiotiques a reçu 8379 visites en janvier 2009. Il est intéressant de noter que $24 \%$ de ces visites proviennent hors de France. Ces chiffres illustrent l'importance de la publication électronique pour valoriser les travaux liés à l'activité scientifique de l'Université de Limoges.

\subsubsection{La numérisation des herbiers}

L'herbier napoléonien de Jean-Baptiste Fray-Fournier conservé au SCD de Limoges est répertorié dans la base de données Sonnerat ${ }^{20}$. Il a été numérisé planche par planche et il est

\footnotetext{
${ }^{16}$ Le dépôt de la thèse est mixte : papier et électronique. La thèse originelle reste la thèse papier

${ }^{17} 575$ thèses sont consultables sur : http://www.unilim.fr/scd/theses/accesdoc.html

${ }^{18}$ Presses Universitaires de Limoges : http://www.pulim.unilim.fr/

${ }^{19}$ Les Nouveaux Actes Sémiotiques sont accessibles à l'adresse suivante : http://revues.unilim.fr/nas

${ }^{20}$ Base Sonnerat : http://coldb.mnhn.fr/colweb/form.do?model=SONNERAT.wwwsonnerat.wwwsonnerat.wwwsonnerat
} 
consultable via un site internet de partage de photos Flickr ${ }^{21}$. Un autre herbier représentant une collection de planches plus importante, l'herbier Le Gendre est en cours de traitement. La description et la numérisation des planches sont effectuées par les bibliothécaires en collaboration avec les chercheurs.

\subsubsection{Le portail Maduvil}

Le portail Maduvil ${ }^{22}$, portail des ressources documentaires du Limousin a pour objet l'interrogation simultanée des catalogues des bibliothèques de l'Université de Limoges et de la Ville de Limoges. La recherche fédérée s'appuie sur le protocole Z 39-50, protocole bien connu des bibliothécaires.

La version $2 \mathrm{du}$ portail Maduvil en cours de réflexion a pour ambition de devenir le portail régional des ressources documentaires et de réunir les collections patrimoniales de toute la région. La recherche sera progressivement élargie à d'autres bibliothèques de la région ainsi qu'aux ressources numérisées dans Gallica concernant le Limousin grâce au moissonnage des données par le protocole OAI-PMH ${ }^{23}$.

Toutes ces réalisations montrent la capacité du SCD de Limoges à former des compétences en interne pour concevoir et organiser la valorisation de la documentation numérique.

\subsection{La formation des usagers AU SCD de Limoges}

L'offre de documentation s'accompagne d'une offre de services : formation des usagers, formation des doctorants, aide à la recherche, service de référence à distance.

La formation est proposée tout au long du cursus et plus particulièrement aux $\mathrm{L}^{24}$, masters et doctorants. Les étudiants de L1 se voient proposer des visites de bibliothèques et une initiation à la recherche dans les catalogues. Une formation à la méthodologie de recherche documentaire, aux outils de veille automatisés et à l'utilisation des ressources électroniques est mise en place pour les masters. Les doctorants sont formés à la rédaction de la bibliographie, aux archives ouvertes ${ }^{25}$ et à l'utilisation de la feuille de style pour la thèse électronique. Des demandes récurrentes sur la formation aux logiciels de gestion de références bibliographiques ont pu être satisfaites notamment par des formations à Zotero ${ }^{26}$.

Toutes ces formations qui sont assurées par des bibliothécaires s'intègrent dans un plan de formation cohérent mis en place par le SCD de Limoges. Un fait marquant mérite d'être signalé puisqu'en 2008, l'évaluation d'étudiants de $\operatorname{master}^{27}$ a été possible avec notation dans le cadre d'un cours de méthodologie de la recherche.

\footnotetext{
${ }^{21} \mathrm{http}: / /$ www.flickr.com/photos/scdlimoges/

${ }^{22}$ Portail Maduvil : http://maduvil.limoges.fr/

${ }^{23}$ Open Archives Initiative - Protocol for Metadata Harvesting

${ }^{24}$ Première année de licence

${ }^{25}$ En 2008, une équipe du SCD a assuré la mise en place du portail HAL pour l'Université de Limoges. HAL-UNILIM : http://halunilim.archives-ouvertes.fr/

${ }^{26}$ Zotero : http://www.zotero.org/

${ }^{27}$ Master LLCE : langues, littératures et civilisations étrangères
} 


\section{Les ressources pédagogiques numériques}

\subsection{Définition des RPN ${ }^{28}$}

Les ressources pédagogiques recouvrent à la fois des outils didactiques, des méthodes d'apprentissage, des contenus et des personnes ressources. On s'intéresse ici plus spécifiquement aux objets pédagogiques qui se présentent soit sous la forme de cours, de plans de cours, d'exercices, d'études de cas, de QCM... Ces objets pédagogiques peuvent être des objets isolés ou alors agrégés dans des ensembles formant un cours, un module voire un cursus préparant à un diplôme.

Au sens de la norme Z 76-040²9, qui décrit le Profil français d'application du LOM (dénommé LOMFR), métadonnées pour l'enseignement, une ressource pédagogique est une ressource utilisée dans un contexte d'enseignement, de formation ou d'apprentissage. Ces ressources sont de formes variées, documents électroniques ou documents physiques : texte, image, vidéo, son, livre, objet, etc...

\subsection{Caractéristiques}

\subsubsection{Du polycopié au cours en ligne}

La transposition électronique par simple numérisation d'un polycopié est la première étape du cours en ligne. L'exposition virtuelle Jean Carbonnier ${ }^{30}$ mise en place par la BIU Cujas propose par exemple un accès intégral aux cours polycopiés numérisés de l'auteur.

A un niveau plus élaboré, il existe des sites proposant des cours complets en ligne. Par exemple, AESplus.net ${ }^{31}$ a vocation à une entraide entre étudiants d'AES $^{32}$ : sur ce site personnel, on trouve des fiches de cours, un forum d'entraide.

Au niveau le plus construit, les ressources pédagogiques recouvrent un ensemble de services, de logiciels et de contenus accessibles dans le cadre d'une activité de formation ou d'apprentissage qui s'agencent dans les plates-formes pédagogiques ou des portails (LMS ou Learning Management System). A l'Université de Limoges, les enseignants déposent leurs cours sur une plate-forme Moodle. Cette plate-forme permet aux étudiants d'accéder à un certain nombre de cours, mais également de déposer des devoirs, y compris en temps limité, d'interroger un enseignant pour demander de l'aide, d'obtenir un suivi et une évaluation

\footnotetext{
${ }^{28} \mathrm{RPN}$ : ressources pédagogiques numériques

${ }^{29}$ ASSOCIATION FRANÇAISE DE NORMALISATION (AFNOR). Technologies de l'information pour l'éducation, la formation et l'apprentissage : profil français d'application du LOM (LOMFR) : Métadonnées pour l'enseignement. NF Z 76-040. 2006

${ }^{30} \mathrm{http}: / /$ expocujas.univ-paris1.fr/Carbonnier/index.html

${ }^{31} \mathrm{http}: / /$ www.aesplus.net/forum/portal.php

${ }^{32} \mathrm{AES}$ : Licence d'Administration économique et sociale
} 
personnalisée. La plate-forme Moodle peut être apparentée à un prolongement du cours en présentiel sous forme d'une classe virtuelle.

\subsubsection{L'intention pédagogique}

L'intention pédagogique est un élément caractéristique de la ressource pédagogique. Une image seule ou une vidéo sans accompagnement ne suffisent pas à constituer une ressource pédagogique. Une description de l'intention pédagogique, c'est à dire du contexte à des fins de formation permet de qualifier la ressource en ressource pédagogique.

\subsubsection{La granularité}

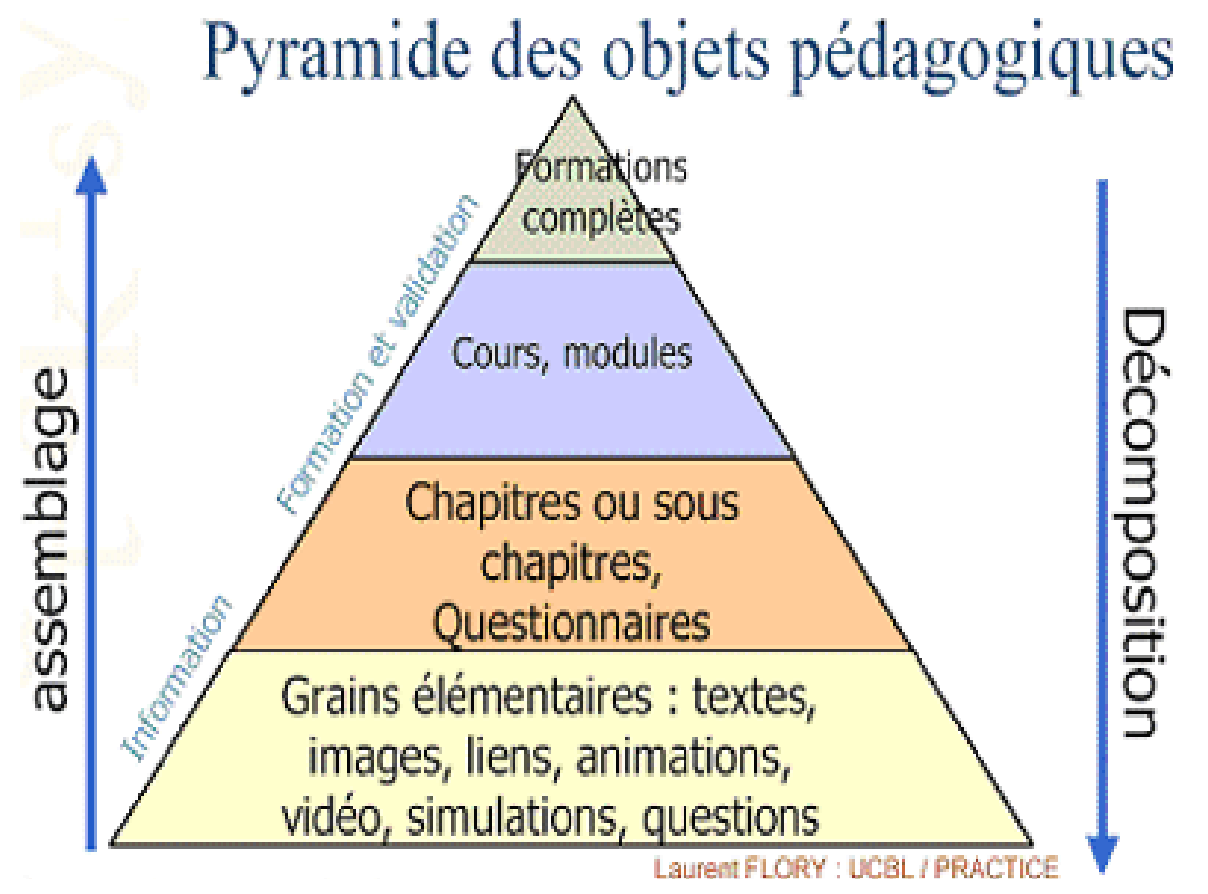

Les caractéristiques d'une ressource pédagogique et les besoins d'indexation qui en résultent. ${ }^{33}$

La granularité est l'une des particularités des objets pédagogiques. Un cours en ligne est de plus en plus souvent un assemblage de textes, d'animations, d'illustrations, de vidéos, de quizz...

De ce fait, le partage des ressources pédagogiques en ligne vise à gagner du temps sur l'élaboration d'un nouveau cours en se consacrant à la valeur ajoutée propre à l'enseignant. Une bonne description de ces cours doit permettre de les retrouver au niveau le plus élémentaire.

La granularité permet aussi d'élaborer des parcours individualisés.

${ }^{33}$ FLORY, Laurent. Les caractéristiques d'une ressource pédagogique et les besoins d'indexation qui en découlent [en ligne]. Disponible sur : $<$ www.enssib.fr/bibliotheque-numerique/document-1234>. (Consulté le 24 août 2010) 


\subsubsection{Les acteurs de la création}

En matière de création de cours en ligne, s'il existe encore des enseignants créant leur propre page web pour y déposer un cours, ce modèle tend à disparaître au profit de chaines de traitement nécessitant plusieurs intervenants :

- le concepteur de la ressource pédagogique : services TICE des universités

- $\quad$ le rédacteur de contenu : enseignants

- le conseil en ingénierie pédagogique pour la conception de scénarios : services TICE des universités

- les équipes informatiques : CRI (centres de ressources informatiques des universités)

- les équipes techniques multimédia, infographistes : services TICE des universités

- $\quad$ les spécialistes de la description et de l'indexation : SCD des universités

Il est important de noter qu'un arrêté du 31 juillet $2009^{34}$ reconnaît comme activité pédagogique le fait d'élaborer et mettre en ligne un module d'enseignement ou de formation, sans tâches directes liées à l'assistance et l'évaluation des étudiants.

\subsubsection{Le coût des ressources pédagogiques}

Même si certains cours sont accessibles librement sur le web, il faut considérer les coûts inhérents à la production d'une ressource numérique.

Dans le cas des ressources d'initiative privée, la gratuité d'accès est compensée par une publicité parfois envahissante et gênante.

L'apparente facilité de déposer et partager des ressources au sein d'une communauté universitaire à un coût direct presque nul ne doit pas masquer les coûts réels en terme de moyens techniques, ressources humaines, ressources techniques.

\subsubsection{Les publics et les usages}

Les ressources pédagogiques en ligne s'adressent autant aux enseignants qu'aux étudiants.

Un cours en ligne peut être utilisé par un enseignant en appui d'un cours en présentiel ou en complément d'un TD. Dans certains cas, il est même vivement recommandé de ne pas utiliser certaines ressources en autoformation. C'est le cas de l'avertissement ci-dessous vu sur le site de Canal- $\mathrm{U}^{35}$ dans le cadre d'un cours sur le droit de la propriété intellectuelle :

\footnotetext{
${ }^{34}$ MINISTERE DE L'ENSEIGNEMENT SUPERIEUR ET DE LA RECHERCHE. Arrêté du 31 juillet 2009 approuvant le référentiel national d'équivalences horaires établi en application du II de l'article 7 du décret n 84-431 du 6 juin 1984 modifié fixant les dispositions statutaires communes applicables aux enseignants-chercheurs et portant statut particulier du corps des professeurs des universités et du corps des maîtres de conférences [en ligne]. Journal officiel, $\mathrm{n}^{\circ} 0187$ du 14 août 2009. Disponible sur : <http://www.legifrance.gouv.fr/affichTexte.do? cidTexte=JORFTEXT000020974583>

${ }^{35} \mathrm{http}: / /$ www.canal-u.tv/
} 
"L'utilisation de ces ressources numériques, qui ne constituent pas un outil d'auto formation, est confiée aux enseignants et aux spécialistes de la propriété intellectuelle qui dispensent les connaissances auprès des étudiants et des acteurs économiques et scientifiques. En effet, le choix, nécessairement arbitraire des thématiques abordées et dont certaines seront rapidement atteintes d'obsolescence, la complexité de certaines notions envisagées et leur approche nécessairement tronquée, rendent indispensable la présentation de tout ou partie de son contenu au public non averti en présence d'un "sachant", bon connaisseur de la propriété intellectuelle. Seule, en effet, la participation active d'un expert du domaine, apte à expliquer, compléter ou simplement traduire les saynètes présentées, permet l'utilisation des ressources dans des conditions pédagogiques optimales. Lui sont dès lors indiqués, de manière très succincte, dans le livret pédagogique annexe : le scénario de chaque saynète, le thème principal abordé et les principaux éléments se rattachant à ce thème. "

Les publics visés par les ressources en ligne sont aussi les étudiants salariés, empêchés, handicapés, en stage à l'étranger, francophones partout dans le monde, en reprise d'études.

Dans un numéro spécial de la revue Études de communication consacré au thème de l'intégration du numérique dans les formations du supérieur, Pierre Moeglin ${ }^{36}$ distingue les trois vies des ressources pédagogiques numériques tout en évoquant la sous-utilisation de ces ressources :

- première vie : la formation en face à face rencontre des difficultés car les enseignants sont réticents à utiliser en cours des ressources pédagogiques qu'ils n'ont pas eux-mêmes conçus

- deuxième vie : l'auto-formation se heurte aux réticences des étudiants qui estiment que le concepteur de la ressource n'est pas celui dont dépend leur réussite à l'examen

- troisième vie : l'autodidaxie qui peut sembler prometteuse reste marginale. De plus, même si elle constitue un " débouché ", ceux qui financent ces ressources ne souhaitent probablement pas se détourner de leur public initial, à savoir le public étudiant.

Pierre Moeglin avance l'idée qu'il est possible de donner " une quatrième vie " à ces ressources par le développement du courtage éducatif. Le courtage éducatif consiste à établir le lien entre l'offre et la demande de connaissances. D'après l'auteur, les ressources pédagogiques numériques projetées hors du cercle de leurs producteurs et utilisateurs souffrent d'une relative sous-exploitation. Faute d'intermédiation, il devient nécessaire d'en inventer.

\subsection{L'indexation des Ressources pédagogiques}

Pour rendre visibles les ressources pédagogiques, il est nécessaire de les indexer. Comme exposé ci-dessus, les ressources pédagogiques présentent des spécificités qui ne rendent pas possibles leur description dans un format de description bibliographique comme MARC ${ }^{37}$. C'est pourquoi des schémas de description ont été développés spécifiquement pour les ressources pédagogiques.

\footnotetext{
${ }^{36}$ MOEGLIN, Pierre. "Le professeur et le courtier : une quatrième vie pour les ressources des campus numériques? " in : "Etudes de Communication ", Numéro spécial, 2007/2

${ }^{37}$ MAchine Readable Cataloging
} 
Le LOM (Standard for Learning Object Metadata ou Recommandation de métadonnées pour des objets pédagogiques) répond à cette nécessité de fournir un schéma de description adapté aux spécificités des ressources à but pédagogique. La description des ressources pédagogiques en LOM permet d'échanger uniquement les métadonnées et facilite l'échange, le partage et la réutilisation de ces ressources pédagogiques.

Le LOM a été conçu en 2002 par le Learning Technology Standards Commitee (LTSC) de l'IEEE (Institute of Electrical and Electronics Engineers). C'est un standard qui s'applique comme une recommandation, il n'a pas la valeur d'une norme. Plusieurs pays dont la France et la Chine se sont opposés à la publication du LOM en tant que norme, car trop marqué par une conception américaine de l'enseignement.

Le LOM propose un schéma de description des ressources pédagogiques en neuf catégories pour neuf fonctions différentes : général, cycle de vie, méta-métadonnées, technique, pédagogique, droits, relation, commentaire, classification. La carte conceptuelle du $\mathrm{LOM}^{38}$ peut être consultée en ligne, elle permet de visualiser l'arborescence complète avec ses 79 éléments.

Parmi les neuf catégories proposées, deux catégories sont essentielles. D'une part, la catégorie pédagogique où l'on retrouve le contexte d'utilisation (université, formation professionnelle, enseignement primaire...), le public visé (apprenant, enseignant), le niveau de difficulté, le temps d'apprentissage, le niveau d'interactivité (de très faible à très élevé). D'autre part, la catégorie classification intéressera plus particulièrement les bibliothécaires. Cette catégorie permet l'utilisation de classifications et de vocabulaires contrôlés. En matière pédagogique, on pourra appliquer des classifications non seulement disciplinaires, mais aussi concernant les compétences ou l'accessibilité. A titre d'exemple, UNIT, l'Université Numérique Ingénierie et Technologie utilise la classification Dewey.

En France, le schéma LOMFR ${ }^{39}$ a été adopté grâce à un partenariat AFNOR-SDTICE ${ }^{40}$. Ce schéma est devenu une norme en 2006 : norme Z 76-040 ${ }^{41}$.

La norme LOMFR a été déclinée en SupLOMFR ${ }^{42}$ pour l'enseignement supérieur par un groupe de travail inter-UNT en $2008^{43}$. On y trouvera des éléments obligatoires et l'ajout de vocabulaires contrôlés adaptés au contexte de l'enseignement supérieur. L'une des caractéristiques de SupLOMFR est d'avoir rendu obligatoire l'utilisation de la classification Dewey. L'outil d'indexation WebDewey actuellement testé par des établissements du réseau ABES pourrait s'avérer intéressant dans ce contexte ${ }^{44}$.

SupLOMFR s'appuie sur les apports du LOMFR tels que la reformulation de listes de valeurs adaptées au contexte français, l'ajout de valeurs spécifiques au système éducatif français (exemple : "démonstration"). SupLOMFR ajoute au profil LOMFR ses propres

\footnotetext{
${ }^{38} \mathrm{http}: / /$ www.lom-fr.fr/cartes

${ }^{39}$ LOMFR : Profil français d'application du Learning Object Metadata

${ }^{40}$ Sous-Direction des Technologies de l'information et de la Communication pour l'éducation. La SDTICE n'existe plus depuis juin 2010. Au sein de la DGESCO (Direction générale de l'enseignement scolaire), une sous-direction reprend les missions de la SDTICE pour l'enseignement du premier et du second degré

${ }^{41}$ ASSOCIATION FRANÇAISE DE NORMALISATION (AFNOR). Technologies de l'information pour l'éducation, la formation et l'apprentissage : profil français d'application du LOM (LOMFR) : Métadonnées pour l'enseignement. NF Z 76-040. 2006

${ }^{42}$ Schéma SupLOMFR en annexe 5 page 54

${ }^{43}$ Guide d'usage de SupLOMFR : http://www.sup.lomfr.fr/index.php?title=Accueil

${ }^{44}$ Expérimentation de la WebDewey : appel à candidature : http://fil.abes.fr/2009/10/19/experimentation-de-la-webdewey-appel-acandidatures/
} 
caractéristiques : des valeurs pour les niveaux d'étude bac +1 , bac +2 , etc.... et l'ajout d'un élément " crédits ECTS ${ }^{45}$ ".

\subsection{Offre de RPN dans les UNT}

\subsubsection{La mise en place des UNT}

Un article de Françoise Thibault ${ }^{46}$ permet de comprendre comment s'est effectuée la mise en place progressive des campus numériques en France. Les premiers appels d'offres en 19992000 ont concerné la construction d'une offre nationale de formation ouverte et à distance (FOAD). L'offre de FOAD a ensuite été réorientée vers une offre plus large à destination de tous les étudiants.

Les universités numériques thématiques sont nées d'une impulsion du Ministère en 2003. L'UMVF (Université Médicale Virtuelle Francophone) a inspiré la création de ces universités numériques thématiques.

En 2010, il existe sept UNT en France qui mutualisent à l'échelle nationale des contenus pédagogiques sur une thématique. Il s'agit de : UNJF (Université Numérique Juridique Francophone), UNF3S (Université Numérique Francophone des Sciences de la Santé et du Sport), UNISCIEL (Université des SCIences En Ligne), UNIT (Université Numérique Ingénierie et Technologie), UVED (Université Virtuelle Environnement et Développement durable), UOH (Université Ouverte des Humanités) et AUNEGE (Association des Universités pour l'enseignement Numérique en Economie-Gestion).

Les UNT ont pour missions de :

- recenser les ressources pédagogiques numériques existantes dans les établissements

- $\quad$ produire de nouvelles ressources

- $\quad$ valoriser, indexer et diffuser ces ressources

- $\quad$ assurer la validation scientifique, pédagogique et technique des ressources produites

Afin de favoriser la réussite en premier cycle, les UNT privilégient la mise à disposition de ressources pour les licences. Il est à noter également que les Universités Numériques Thématiques malgré la dénomination " université " n'inscrivent pas les étudiants, ne valident pas de formation et ne délivrent pas de diplômes.

Un portail " Universités numériques "47 a été mis en place en 2008 pour offrir un accès unique aux ressources des UNT. La recherche fédérée sur les ressources des différentes UNT est possible grâce au protocole OAI-PMH.

\footnotetext{
${ }^{45}$ ECTS : European credit transfer system, système européen de transfert et d'accumulation de crédits

${ }^{46}$ THIBAULT, Françoise. Autour des campus numériques français : Repères dans les initiatives du ministère en charge de l'enseignement supérieur in : Distances et Savoirs, 2006/1, Vol. 4, p. 109-112

${ }^{47} \mathrm{http}: / /$ www.universites-numeriques.fr/
} 


\subsection{2 les UNT et leurs spécificités}

Si la référence à une discipline et la mutualisation des ressources constituent des points communs aux UNT, la réalité est plus hétérogène. Les structures varient, les modèles économiques divergent et les choix techniques sont variés.

Les statuts des UNT sont très variables. UNJF et UNF3S sont des groupements d'intérêt public $(\mathrm{GIP})^{48}$, AUNEGE et UVED se sont constitués en association, UNISCIEL est un groupement d'intérêt scientifique (GIS). UNIT a adopté un statut de fondation et UOH est régi par un service commun inter-universitaire.

Chaque UNT est dirigée par une instance collégiale décisionnaire avec un président, un directeur et un conseil d'administration. Les UNT engagent des partenariats avec les universités. Celles-ci deviennent adhérentes avec une contribution financière dans le but d'accéder aux ressources ou alors elles deviennent partenaires associés dans le but de collaborer à des projets.

Certaines UNT donnent accès librement et gratuitement à leurs ressources ou une partie de leurs ressources sur le web. D'autres ont choisi un modèle économique payant. Dans ce cas, le coût est supporté par les universités adhérentes ${ }^{49}$.

Les politiques éditoriales des UNT sont variables. Certaines restent assez fidèles aux programmes telles l'UNJF et AUNEGE. A l'inverse, UOH cherche plutôt à se positionner comme une " bibliothèque inter-universitaire publique " en proposant à la fois des cours, des conférences ou des entretiens. UNF3S regroupe sur un même portail plusieurs campus thématiques : médecine, pharmacie, odontologie, sciences du sport.

Les ressources créées pour les UNT peuvent être l'objet de développement multimédia très soignés : vidéos, simulation de TP pour UNISCIEL, études de cas pour AUNEGE alors que les ressources de l'UNJF sont essentiellement textuelles.

Les ressources des UNT sont moissonnables à condition d'avoir été indexées dans un format compatible avec le protocole OAI-PMH. Quelques UNT ont recruté des documentalistes pour indexer leurs ressources : c'est le cas d'UNIT et UNISCIEL. Inversement, une UNT peut moissonner des ressources indexées par les universités.

\subsubsection{UNR et UNT}

Les UNT ont été mises en place avec la volonté d'offrir des contenus labellisés. En parallèle du développement des UNT, les Universités Numériques en région (UNR) ont été mises en place pour offrir des services numériques : accès aux $\mathrm{ENT}^{50}$, carte multi-services, accès aux ressources. Ainsi, une UNR peut soutenir financièrement un ou plusieurs établissements ayant un projet autour de l'accès aux ressources pédagogiques.

\footnotetext{
${ }^{48} \mathrm{GIP}$ : groupement d'intérêt public

${ }^{49} \mathrm{~A}$ titre d'exemple, une université ayant moins de 1200 étudiants inscrits dans une formation comportant du droit ou des sciences politiques verse une contribution annuelle de $5000 €$ à l'UNJF, tandis qu'une université comptant 3500 étudiants cotise à hauteur de $10000 €$ par an (chiffres 2010)

${ }^{50}$ Environnement Numérique de Travail
} 
L'Université de Limoges membre de l'UNR-PCL Poitou-Charentes-Limousin doit pouvoir compter sur un appui pour un projet visant à faciliter l'accès aux ressources pédagogiques.

\subsection{LES ENJEUX}

La réussite des UNT tient tout d'abord au fait de rendre visible le patrimoine pédagogique des universités, de faciliter ainsi l'accès aux ressources et le travail des étudiants donc la réussite étudiante.

La visibilité des ressources vise aussi à assurer la promotion des universités françaises dans un contexte international : être publié dans une UNT est promotionnel à l'échelon national et international ${ }^{51}$. Le rapport ISAAC ${ }^{52}$ préconise d'insérer les établissements d'enseignement supérieur dans les réseaux numériques de la connaissance afin de répondre à l'enjeu de leur présence à l'international.

Pourtant, selon une enquête menée en 2010 par une étudiante de master à Lille 3 pour le compte d'UNIT, les UNT sont largement méconnues des étudiants ${ }^{53}$. On leur reproche d'être déconnectées des universités. L'article de Pierre Moeglin déjà cité ${ }^{54}$ signale aussi une relative sous-utilisation des UNT.

On peut relativiser ce point de vue grâce à un article d'Elisabeth Fichez ${ }^{55}$ sur la notion de réussite et d'échec des campus numériques. Que signifie réussite ou échec d'un campus numérique. Peut-on mesurer la réussite ou le relatif échec uniquement à la fréquentation de la plate-forme. Est-ce un échec financier? Certains pensent que les moyens financiers investis sont nettement insuffisants pour assurer la réussite totale des UNT. Au crédit des UNT, on peut attribuer la mutualisation et la qualité des ressources produites.

La réussite des UNT s'illustre aujourd'hui par la production de ressources labellisées avec une mise à jour garantie. Les UNT s'engagent à produire des ressources à haute valeur ajoutée. La qualité scientifique et pédagogique est assurée par les conseils scientifiques qui sélectionnent, expertisent et valident les ressources.

La qualité technique de la production est également garantie grâce à la mise en place d'outils telles les chaines éditoriales et une bonne indexation des ressources. Des groupes de travail inter-UNT ont été mis en place pour accompagner la production et la diffusion des ressources. Ils sont organisés en quatre thématiques : chaînes éditoriales, indexation, ORI-OAI ${ }^{56}$, questions juridiques.

Les moyens financiers que peuvent apporter les UNT permettent des appels à projets de qualité. Des moyens financiers pour " scénariser " les cours se justifient car la seule approche

\footnotetext{
${ }^{51}$ Entretien avec le Professeur Albert-Claude Benhamou, promoteur des UNT in : Distances et savoirs, Vol. 4, n²006/1, p. 99-107

${ }^{52}$ ISAAC, Henri. L'Université numérique : rapport à Madame Valérie Pécresse, Ministre de l'enseignement supérieur et de la recherche. 2008 [en ligne]. Disponible sur : <http://media.enseignementsup-

recherche.gouv.fr/file/Nouvelle_universite/55/7/universitenumerique_23557.pdf\$. (Consulté le 24 août 2010)

${ }^{53}$ Les résultats de cette enquête menée par Cécile Pouliquen en master 1 Sciences de l'information et du document à Lille 1 ont été présentés lors des journées CIUEN 2010 à Strasbourg mais ne sont pas encore diffusés

${ }^{54}$ MOEGLIN, Pierre. Le professeur et le courtier : une quatrième vie pour les ressources des campus numériques? in : Etudes de Communication, Numéro spécial, 2007/2

${ }^{55}$ FICHEZ, Elisabeth. Campus numériques français : pertinence des notions de réussite et d'échec. in : Etudes de Communication, Numéro spécial, 2007/2

${ }^{56} \mathrm{ORI}-\mathrm{OAI}$ : Outil de Référencement et d'Indexation compatible OAI-PMH
} 
réduite à la fourniture de contenu ne permet pas d'utiliser tout le potentiel de communication des services que permet le Web. Une étude norvégienne ${ }^{57}$ montre comment la technologie est un catalyseur de changement et entraine des innovations qui vont bien au delà de la conception des TIC comme simple outil.

${ }^{57} \mathrm{JOPP}$, Karsten et TREBBI, Turid. Campus numériques : quel impact sur l'enseignement supérieur en Norvège ? in : Distances et savoirs 2006/1, Volume 4, p. 13-22 


\section{Des applications concrètes}

\subsection{Des projets autour des ressources pédagogiques}

\subsubsection{Le projet indexation des ressources pédagogiques au SCD Paris-Sud 11, Orsay}

Deuxième université française citée au classement de Shanghai, l'Université de Paris-Sud 11 est une université ayant une réputation d'excellence. Elle compte environ 27000 étudiants avec une longue tradition d'ouverture à l'international.

Dans son rapport d'activité 2009, l'Université Paris-Sud 11 met en avant parmi ses projets visant à aider à la réussite des étudiants, l'important développement des TICE avec le recensement des ressources pédagogiques produites par l'université, la production de modules de formation originaux et l'indexation de ces ressources par le SCD. Par ailleurs, le rapport souligne que l'attractivité des formations de l'université se ressent dans une augmentation de plus de $10 \%$ des effectifs étudiants à la rentrée 2009 .

Le groupe projet " ressources pédagogiques " de l'Université comprend cinq personnes : deux au service TICE, une au CRI, et deux au SCD.

$\mathrm{Au}$ sein du SCD, Catherine Weill, conservateur des bibliothèques et correspondant ressources pédagogiques a apporté des éléments d'information lors d'un entretien téléphonique mené en mars 2010 .

Elle précise d'emblée qu'une collaboration des services TICE, CRI et SCD est nécessaire pour la réussite du projet.

Le projet repose techniquement sur le choix d'une plate-forme Dokeos (LMS) pour le dépôt institutionnel $^{58}$ et la mise en place du logiciel ORI-OAI. Le paramétrage d'ORI-OAI a été mené par un informaticien. Compte tenu du temps investi en paramétrage, il est à noter qu'un projet mené à l'échelle d'une UNR aurait pu constituer un meilleur choix.

Un ingénieur d'études assure l'indexation des ressources selon le schéma SupLOMfr, schéma préconisé par le Ministère. Une articulation avec l'UNT Unisciel se fait par moissonnage des métadonnées, d'où l'importance que ces métadonnées soient établies selon la norme et que les entrepôts OAI soient correctement configurés.

L'étape d'indexation est donc une étape importante du workflow où le SCD joue un rôle majeur. Si l'auteur est en mesure de fournir un minimum de métadonnées, il faut être conscient que les enseignants sont généralement peu disposés à passer du temps pour renseigner ces métadonnées. D'où l'importance du recrutement de l'IGE ${ }^{59}$ dont le poste a été repris au compte du SCD et qui assure ce travail d'indexation. Cependant, le contrat est à

\footnotetext{
${ }^{58}$ Une partie des cours de la plate-forme Dokeos sont en accès libre : http://formation.u-psud.fr/open_courses.php

${ }^{59}$ Ingénieur d'études
} 
durée limitée et pose le problème de la suite à donner. Il est possible d'envisager d'automatiser les médatonnées techniques, mais tout ne peut pas être automatisé et le reste ne peut pas être intégralement fait par les bibliothécaires. Il faut compter environ $1 / 2$ heure par ressource. Par ailleurs, le schéma SupLOMFR peut être vu comme contraignant. Le guide d'usage de SupLOMFR disponible en ligne ${ }^{60}$ est suffisant s'il est complété d'une formation telle que celle proposée par l'ENSSIB sur l'indexation des ressources pédagogiques.

Enfin, le niveau de granularité de l'indexation au niveau le plus élémentaire (un texte, une image, une vidéo, une séquence de vidéo), niveau d'indexation recommandé dans la théorie s'avère difficile à appliquer car les cours se présentent en "espace de cours" avec des liens entre eux.

La visibilité des ressources pédagogiques, selon le choix de l'Université de donner un accès unique à toutes les ressources documentaires, pédagogiques et littérature grise, est assurée par le SID $^{61}$ du SCD au travers d'un portail Archimed dont l'ouverture prévue initialement pour mai 2010 a été repoussée à septembre $2010^{62}$.

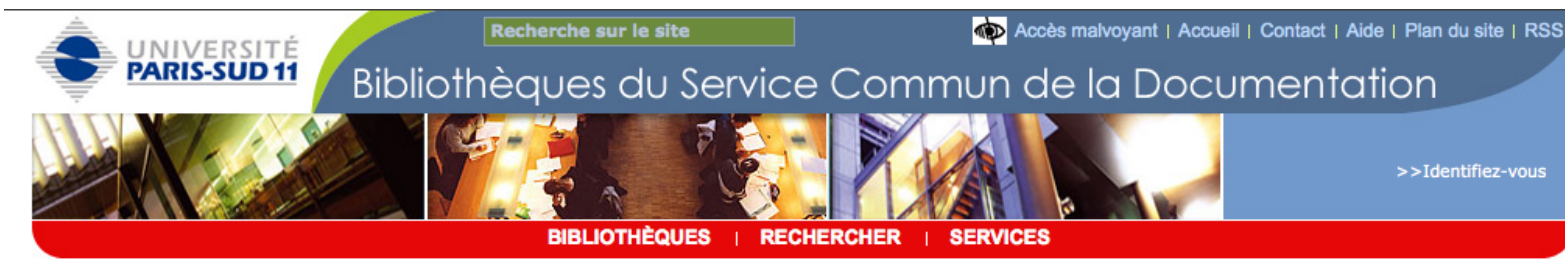

Accueil $>$ Rechercher $>$ Production de Paris-Sud $11>$ Cours en ligne

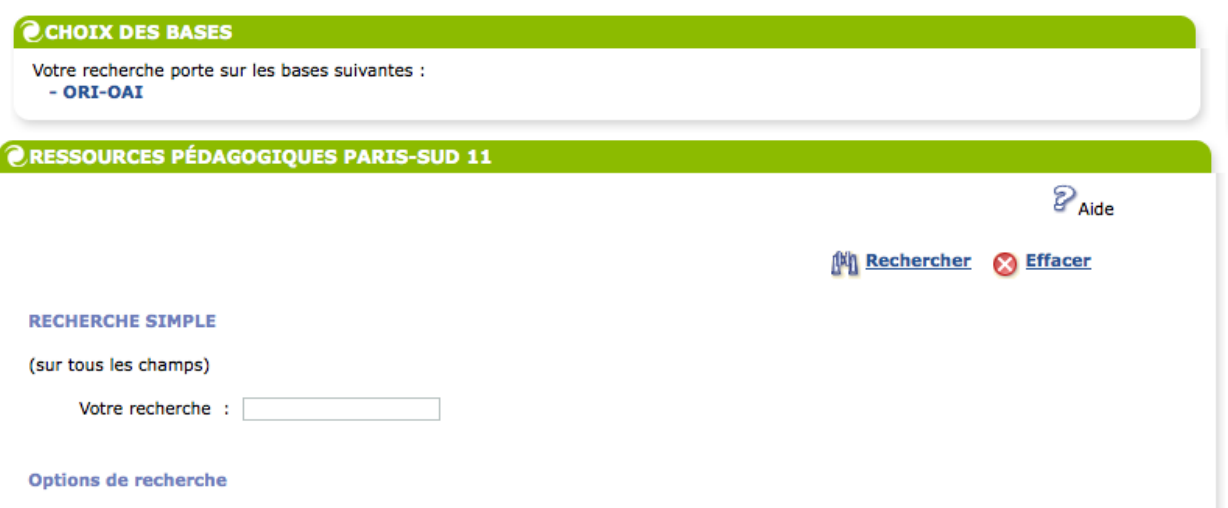

Portail du SID de I'Université Paris-Sud 11, Orsay version en test

En conclusion, Catherine Weill insiste sur l'enjeu d'un tel projet pour le SCD car il redonne du sens au métier de bibliothécaire au sein de son Université.

\footnotetext{
${ }^{60} \mathrm{http}: / /$ sup.lomfr.fr/index.php?title=Accueil

${ }^{61}$ Système d'Information Documentaire

${ }^{62} \mathrm{http} / / /$ sid.u-psud.fr/
} 


\subsubsection{Université du Maine : un projet porté par l'UNR}

L'Université du Maine est une université de taille plus modeste qui compte environ 10000 étudiants. Le tassement des effectifs étudiants ayant été significatif dans les dernières années, le maintien de ces effectifs est un enjeu qui détermine la politique de l'université ${ }^{63}$.

Le projet de diffusion des ressources pédagogiques de l'Université du Maine a été présenté à plusieurs reprises d'abord en juin 2009 aux journées de l'ABES ${ }^{64}$, puis en juin 2010 lors du Colloque international de l'Université à l'ère du Numérique, CIUEN.

La présentation au CIUEN a été faite par Nicolas Postec et Jennifer Wolfarth de l'Université du Maine. Cette présentation a été complétée par un entretien téléphonique avec Jennifer Wolfarth en juillet 2010.

Ce projet s'inscrit dans le cadre de l'UNR Pays de Loire ${ }^{65}$. Il est mené par la cellule TICE de l'Université du Maine.

Le constat de départ a été la présence d'un important stock de ressources pédagogiques : environ 2000 qui avaient été produites pour l'UVPL (Université Virtuelle des Pays de Loire) en grande partie pour un dispositif de FOAD. Ces ressources pouvaient être utiles aux étudiants en dehors du dispositif d'enseignement à distance mais restaient invisibles. L'idée a été de mettre en place un catalogue des ressources pédagogiques numériques pour rendre ces ressources accessibles.

Avec le soutien de l'UNR Pays de Loire, un chargé de mission a été recruté pour ce projet. Au total, quatre personnes travaillent sur ce projet depuis mars 2008 : une au CRI, deux au SCD et une au service TICE.

Le choix technique repose sur l'outil ORI-OAI pour l'indexation d'une part et sur le SIGB Flora d'autre part pour la recherche et l'affichage. Les ressources sont indexées en SupLOMfr et un guide d'indexation en SupLOMFR a été rédigé à usage interne. Le SCD intervient dans le workflow pour indexer les ressources en Dewey et Rameau.

Le portail documentaire Flora ${ }^{66}$ comporte une fonctionnalité de moissonneur qui a pu être exploitée grâce aux compétences d'un personnel du SCD. Les ressources référencées par ORIOAI sont donc moissonnées par le $\mathrm{SID}^{67}$. Cependant, il n'a pas été possible de mettre en place une recherche fédérée catalogue plus ressources moissonnées. Un formulaire de requête a été développé en XSL avec un moteur de recherche adapté spécifiquement aux ressources pédagogiques.

Le projet s'est déroulé sur 2 ans et demi : la première année a fait l'objet d'un inventaire des ressources produites par l'Université, de l'installation et du paramétrage des outils. La deuxième année a été une année de production des fiches SupLOMFR, perfectionnement des paramétrages, articulation avec le SID, contacts avec les enseignants pour régler la question des droits.

\footnotetext{
${ }^{63}$ Agence d'évaluation de la recherche et de l'enseignement supérieur (France) . Rapport d'évaluation de l'Université du Maine - Le Mans. 2007 [en ligne]. Disponible sur : <http://www.aeres-evaluation.fr/content/download/11297/169776/file/AERES-S1-LeMans.pdf> (consulté le 30 août 2010)

${ }^{64}$ AGENCE BIBLIOGRAPHIQUE DE L'ENSEIGNEMENT SUPERIEUR. L'indexation des ressources numériques en SupLOMFR dans ORI-OAI : un exemple de travail collaboratif à l'université $d u$ Maine 2009 [en ligne]. Disponible sur : $<$ http://www.abes.fr/abes/page,395,journees-abes.html $>$ (consulté le 30 août 2010)

${ }^{65} \mathrm{UNR}$ Pays de Loire : Angers - Nantes - Le Mans

${ }^{66} \mathrm{http}: / /$ catalogues-bu.univ-lemans.fr/flora_umaine/servlet/LoginServlet? profile=anonymous\&success=jsp/system/win_main.jsp\&failure=jsp/error.jsp

${ }^{67}$ Noter que l'INSA de Lyon a choisi la solution inverse : moissonnage des données FLORA vers ORI-OAI
} 
Au final, 900 ressources ont été indexées, 400 ressources libres, 300 ressources de l'UNR et 100 de l'université du Maine. A quelques mois de la mise en production prévue en septembre 2010, les responsables de ce projet exposent les plus et les moins de leur projet lors de leur présentation au CIUEN 2010.

La question de la gestion des droits d'auteur reste encore à régler pour un certain nombre de ressources ainsi que des interrogations sur la "durabilité " d'une ressource et son intérêt pédagogique.

Par ailleurs, un partenariat avec l'UNT UVED a permis de créer des ressources pédagogiques. Ces ressources pédagogiques référencées et indexées sont donc moissonnables par UVED.

Ce projet offre l'avantage d'articuler un portail documentaire avec ORI-OAI ${ }^{68}$ en faisant le choix de présenter les ressources pédagogiques dans un environnement que connaissent déjà les étudiants.

Enfin, il est intéressant de noter que le temps de travail consacré à ce projet par le personnel du SCD est pris en compte par l'UNR.

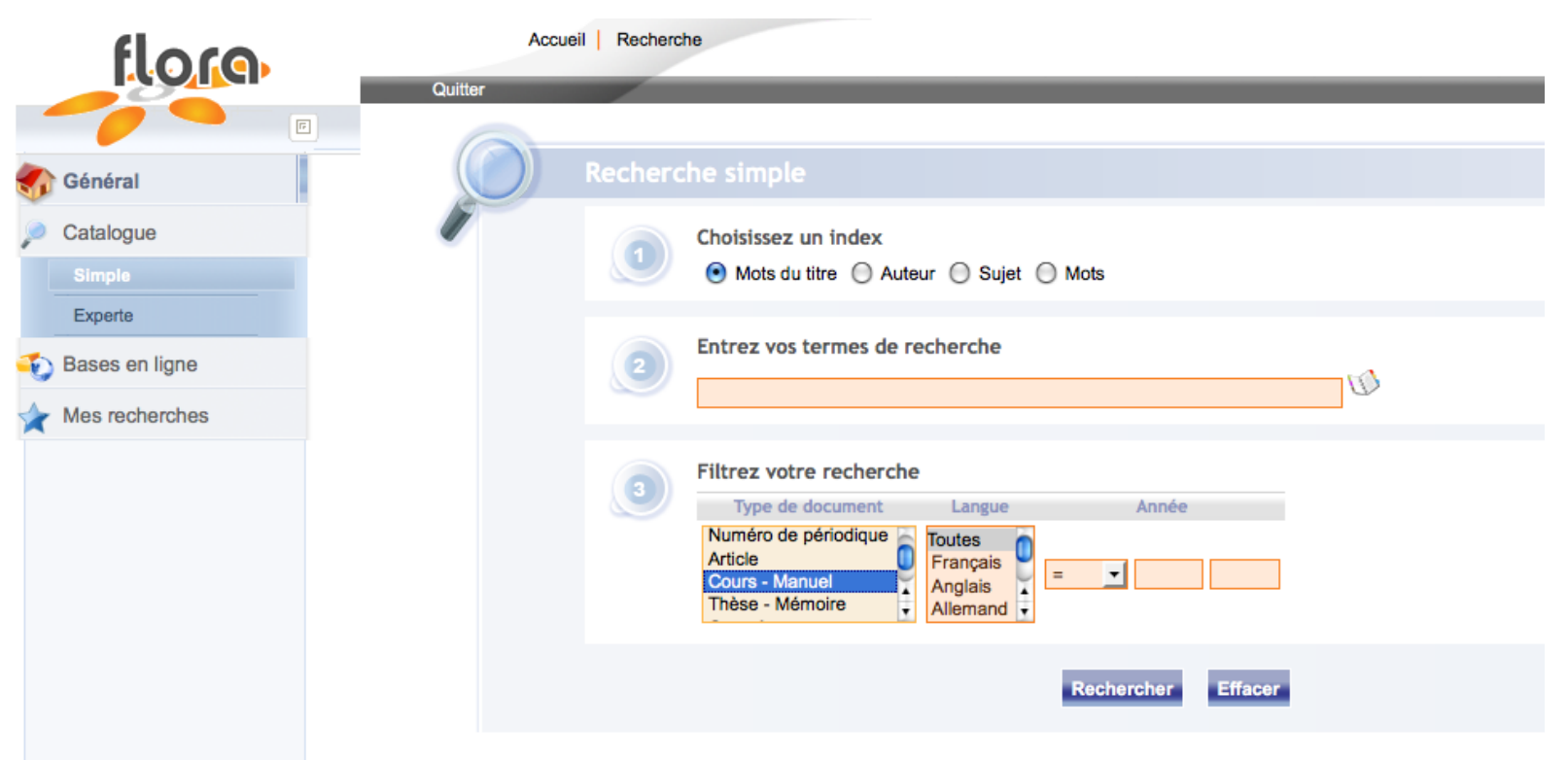

Portail Flora du SCD Le Mans

\subsubsection{IUT en ligne: augmenter les usages par le cartable numérique}

Le projet " IUT en ligne "69 proposé par l'Association des Directeurs d'IUT a été retenu en réponse aux appels d'offre " campus numérique 2000 " du Ministère de l'Education Nationale. Le but de ce projet était la mise à disposition sur le web d'une médiathèque de ressources

${ }^{68}$ L'INSA de Lyon articule également son SIGB Flora avec ORI-OAI mais c'est ORI qui moissonne les données de Flora. L'interface à consulter est donc celle d'ORI

${ }^{69} \mathrm{http}: / / w w w . i u t e n l i g n e . n e t /$ 
pédagogiques à destination des étudiants de DUT et licences professionnelles. A la différence des UNT, ce campus numérique couvre non pas un domaine disciplinaire mais toutes les spécialités des IUT, soit 26 spécialités.

Aujourd'hui, la plate-forme IUT en ligne offre :

- une mutualisation des ressources pédagogiques créées par des enseignants d'IUT, soit 838 ressources dont $70 \%$ sont accessibles librement

- $\quad$ un espace d'échange sous forme d'un forum

- un service d'ingénierie pédagogique

L'une des particularités d'IUT en ligne est de proposer un outil nommé " cartable virtuel ". L'objet de cet outil est de permettre à un enseignant de composer un ensemble de ressources pédagogiques pour les proposer à un étudiant ou un groupe d'étudiants. Ainsi, l'enseignant devient prescripteur d'une ressource pédagogique qu'il n'a pas forcément conçu lui-même mais choisit les ressources qu'il juge adaptées en complément de son cours.

L'exemple d'IUT en ligne est un cas un peu à part car cette ressource n'est pas considérée comme une université numérique thématique. Cependant, IUT en ligne est souvent cité aux côtés des UNT au même titre que Canal $U$, la vidéothèque numérique de l'enseignement supérieur $^{70}$. Les ressources d'IUT en ligne sont indexées et moissonnées sur le portail des Universités Numériques.

L'un des responsables d'IUT en ligne, Serge Paronneau est un enseignant de l'Université de Limoges. Il est également chargé de mission " ressources pédagogiques " pour l'Université de Limoges. Une rencontre avec ce responsable a permis d'éclairer le rôle prescripteur que peut jouer un enseignant ou même un bibliothécaire. La granularité des ressources pédagogiques trouve là tout son intérêt puisque l'on peut prescrire à un étudiant un parcours personnalisé selon ses besoins.

\subsection{Les OBstacLes}

Les obstacles rencontrés dans la mise en oeuvre de projets autour des ressources pédagogiques sont d'ordre réglementaires, financiers, humains, organisationnels.

\section{- obstacle à la production}

Droit d'auteur, plagiat, difficultés techniques, réticences des enseignants à mettre leur cours en accès libre sur internet sont souvent évoqués par les responsables de projets.

\section{- $\quad$ obstacle à la diffusion et à l'utilisation}

Il existe des risques liés à la production de ressources déconnectées de leurs usages et donc vouées à une mise au placard. On peut dès lors s'interroger sur l'écart entre ce qui est prévu par les concepteurs du dispositif, relayé par les prescripteurs, et les pratiques effectives des utilisateurs finaux. Le différentiel se mesure et peut révéler des difficultés à appréhender l'outil ou de mauvaises conditions d'utilisation.

${ }^{70}$ http://www.canal-u.tv/ 


\subsection{Les ENSEIGNEMENTS}

Un stage de l'ENSSIB sur les ressources pédagogiques venu en complément de ces expériences de terrain permet de dégager des enseignements.

Pour les personnels des SCD, la nécessité de convaincre les services TICE, CRI et SCD de travailler ensemble implique de " sortir " de l'établissement, de s'intégrer dans les instances de décision des UFR pour apprendre ce qu'ils produisent et pour mettre en place les partenariats.

Il devient également nécessaire de connaître de nouveaux outils, d'appréhender de nouvelles normes. Il ressort par exemple des besoins de formation des personnels tels les catalogueurs ayant l'habitude de décrire des ressources documentaires pour assurer le passage du catalogage traditionnel vers le catalogage en XML.

Il apparaît aussi une nécessité de médiation, d'interaction ou de référents pour assurer la promotion des UNT. Ces actions de médiation qui demandent des aptitudes pédagogiques impliquent aussi de nouveaux besoins de formation pour les personnels du SCD.

De fait, les "angles d'attaque" sont multiples pour les personnels des SCD et doivent permettre d'assurer une présence là où les compétences des bibliothécaires sont reconnues.

On peut citer par exemple la participation au recensement des ressources pédagogiques déjà existantes avec mise en place d'une veille, la mise à jour des bibliographies ou la localisation des documents proposés aux étudiants dans les cours des UNT, l'expertise sur la méthodologie de recherche qui fait l'objet de cours dans les UNT, la formation des étudiants à l'utilisation de ces ressources, le référencement des ressources elle-même, et enfin l'expertise sur l'archivage des ressources pédagogiques. On pourrait citer aussi l'expertise sur les opérations de rétroconversion puisqu'il existe une problématique de la rétroconversion des ressources pégagogiques déjà existantes avec récupération des données stockées hors de l'université. Enfin, la démarche doit s'accompagner d'une redéfinition des postes qui prend en compte ces nouvelles fonctions. 



\section{Scénarios pour une aide à la décision}

\subsection{Préalables}

Le préalable à un projet " ressources pédagogiques numériques " est d'inscrire en clair cette mission dans les missions du SCD et d'y affecter des moyens humains, financiers et techniques. Selon les scénarios, ces moyens seront plus ou moins importants, mais il convient d'afficher la volonté politique et stratégique de l'établissement concernant la valorisation des ressources pédagogiques comme l'une des missions du SCD. Cette mission doit être portée par le Conseil de la Documentation de l'Université qui se prononce sur la politique documentaire du SCD de Limoges.

Par ailleurs, quel que soit le scénario choisi, il faut être conscient que le projet s'appuiera sur des équipes mixtes où l'on retrouvera des personnels du Centre de Ressources et d'Innovation Pédagogiques CRIP, le chargé de mission " ressources pédagogiques ", des personnels du service commun informatique (SCI), des enseignants et des personnels du SCD de l'Université de Limoges.

Trois scénarios ont été envisagés, chacun d'entre eux contribuant à donner de la visibilité aux ressources pédagogiques des UNT. Les angles d'attaque sont très différents puisqu'on agira soit en aval en moissonnant des métadonnées déjà existantes, soit très en amont de la production des ressources en devenant référenceur-indexeur, soit comme intermédiaire en devenant prescripteur de ressources pédagogiques.

\subsection{ScÉnario 1 : Le SCD moissonneur de Ressources PÉDAGOGIQUES}

\subsubsection{Description et objectifs}

Dans ce scénario, il s'agit de mettre en oeuvre une solution technique de moissonnage des métadonnées des UNT pour les ramener vers le portail du SCD. Le SCD connait la technique du moissonnage OAI grâce à la mise en oeuvre du portail " Maduvil " qui fédère le catalogue du SCD avec celui de la Bibliothèque Francophone Multimédia (BFM) de Limoges. La version 2 de Maduvil ${ }^{71}$ mettra en oeuvre le moissonnage de notices et proposera l'accès à des ressources moissonnées telles celles de Gallica concernant le Limousin. De ce fait, un moissonneur sera installé et des compétences acquises par le personnel.

${ }^{71} \mathrm{http}: / /$ maduvil.limoges.fr/ 


\subsubsection{Mise en oeuvre}

Ce projet peut être porté par la cellule informatique qui est en cours de constitution au SCD de Limoges. L'équipe projet est donc constituée de personnels du SCD dont certains auront déjà acquis des compétences techniques dans la réalisation d'autres projets (numérisation, moissonnage, langage XML, ORI-OAI). Cependant, il faut être vigilant à ne pas limiter le projet à ses aspects techniques. L'intégration d'un outil de recherche au portail actuel du SCD doit être pensée en tenant compte des besoins des usagers.

Le projet moissonnage passe par les étapes suivantes :

- $\quad$ recenser les UNT qui proposent les métadonnées moissonnables : à l'heure actuelle UNIT et UNISCIEL. Mais d'autres UNT comme UVED sont en cours d'installation ou de réflexion ${ }^{72}$.

- $\quad$ étudier les données interrogeables, les sets proposés pour définir des index. A priori, sont interrogeables les index auteur, titre, résumé et mots-clés. Un moissonneur peut être configuré pour moissonner plusieurs entrepôts à la fois.

- $\quad$ se doter d'un outil de moissonnage pour récupérer les métadonnées produites par les UNT et les stocker sur un serveur. Planifier une mise à jour régulière et automatique

- $\quad$ rendre interrogeables les données moissonnées par moteur de recherche

- $\quad$ intégrer la recherche de ressources pédagogiques au portail du SCD soit par un onglet supplémentaire, soit par un choix dans une liste déroulante

- $\quad$ étudier la possibilité d'une recherche fédérée : catalogue et base OAI des ressources pédagogiques

Dans ce scénario, il est possible d'utiliser le logiciel ORI-OAI en mettant en production seulement les modules ORI-OAI nécessaires. Le module ORI-OAI-harvester correspond au moissonneur. Il permet le moissonnage de fiches de métadonnées sur tout entrepôt OAI. Le module ORI-OAI-repository se charge de l'exposition des fiches moissonnées et le module ORI-OAI-indexing indexe les fiches de métadonnées avec le moteur d'indexation Lucene. ${ }^{73}$

D'autres solutions existent :

- des solutions libres mais on perd l'avantage du soutien de la communauté ORI-OAI, communauté qui est très active dans l'enseignement supérieur

- des solutions propriétaires qui contraindront le SCD au choix d'un prestataire et risquent de l'enfermer dans des choix techniques non souhaités

- $\quad$ certains SIGB disposent également d'un module de moissonnage ${ }^{74}$

\footnotetext{
${ }^{72}$ Profil OAI sur le site http://www.ori-oai.org/pages/viewpage.action?pageId=19857423

${ }^{73}$ BOURGES, Raymond. COLMANT, Yohan. HUET, Nolwen. JANNIN, François. L'architecture ORI-OAI in : Archivage et stockage pérennes : enjeux et réalisations. 2009

${ }^{74}$ Liste des clients OAI ou des systèmes incluant un client OAI établie par TOSCA Consultants au 1er janvier 2010 http://www.toscaconsultants.fr/logiciel.htm\#typologie
} 


\subsubsection{Impact et évaluation}

L'impact de cette solution pourrait être assez rapide, avec un investissement moindre puisque l'on s'appuie sur des ressources déjà indexées. Cependant, se pose le problème des ressources des UNT qui ne sont pas actuellement interrogeables par le protocole OAI-PMH comme celles de l'UNJF, AUNEGE, UOH, $\mathrm{UMVF}^{75}$. Bien que fortement incité à indexer leurs ressources en SupLOMFR par le Ministère, il n'est pas sûr que ce soit une priorité pour les UNT concernées.

L'intérêt de ce scénario en particulier avec la mise en route d'ORI-OAI réside dans l'anticipation par rapport à une deuxième tranche qui pourrait être envisagée et conduirait au deuxième scénario.

L'évaluation du projet devrait se baser sur les statistiques d'usage du portail si toutefois il est possible d'obtenir des statistiques assez détaillées sur les parcours des usagers.

\subsection{Scénario 2 : Le SCD réfÉrenceur et indeXeUr de RESSOURCES PÉDAGOGIQUES}

\subsubsection{Description et objectifs}

Ce modèle est inspiré des exemples mis en place à l'Université Paris-Sud 11, Orsay et celle du Maine. Comme exposé au paragraphe 3.1, ces deux établissements ont mené un projet de référencement des ressources pédagogiques produites par leur université ou leur UNR. Le référencement et l'indexation de ces ressources sont effectués dans ORI-OAI. Dans les deux exemples choisis, l'accès aux ressources est possible dans le portail du SCD et les ressources indexées sont moissonnables par les UNT. C'est ainsi que certaines ressources de l'Université d'Orsay sont moissonnées par UNIT, et celles du Mans par UVED. L'élément intéressant est que les deux SCD de ces universités ont su l'un et l'autre rester des acteurs visibles en intégrant le signalement des ressources pédagogiques à leur portail documentaire.

Dans le scénario qui suit, l'accent est mis sur l'indexation en amont dès la production de la ressource. C'est une façon de valoriser la production pédagogique de l'université, de lui donner une visibilité soit locale, soit nationale voire internationale au travers d'une UNT.

\subsubsection{Mise en oeuvre au SCD de Limoges}

La mise en oeuvre de ce scénario passe par un groupe de projet réunissant des personnels du service informatique de l'université, du service des TICE et du SCD. Ce groupe de projet pourrait être composé d'un ou deux représentants de chaque service. L'équipe doit réunir des compétences informatiques, documentaires, pédagogiques et aussi juridiques, ce qui pourrait amener à solliciter des enseignants de l'UFR de droit.

${ }^{75}$ L'UMVF déclare : " Nous devons par ailleurs évoluer vers la norme LOM.fr pour l'intégration d'OAI-PMH sur les données de Doc'UMVF, ce processus visant à faire de Doc'UMVF un entrepôt OAI moissonnable " source http://www.umvf.org/ 
Le chef de projet doit avoir une vision à la fois politique puisque les choix vont impacter sur l'attractivité et la réputation de l'Université, une vison stratégique qui inclut la conduite du changement, la mise en oeuvre des compétences techniques, la clarification des responsabilités, et une vision technologique témoignant de la capacité à suivre l'évolution technologique, enfin une vision à moyen et long terme pour l'intégration du projet dans un $\mathrm{SIG}^{76}$.

Dans les grandes lignes, le projet passe par les étapes suivantes :

- $\quad$ procéder à l'inventaire du patrimoine pédagogique de l'université : dans et hors l'université. On a vu que les ressources pédagogiques peuvent être déposées sur la plateforme Moodle mais il existe aussi certainement des ressources dispersées ailleurs sur des pages internes de l'université ou des sites personnels d'enseignants

- $\quad$ mettre en place la veille et organiser le signalement de ces ressources en s'insérant dans les instances de décision des UFR pour connaître leur production et pour convaincre les interlocuteurs

- se doter d'un outil de référencement et d'indexation : ORI-OAI est le choix probablement le plus judicieux car déjà en place dans d'autres établissements. On pourra donc bénéficier de leur expérience sachant qu'il existe une " communauté " d'experts autour du logiciel ORI-OAI

- $\quad$ mettre en place le workflow : pour le workflow, on peut esquisser un schéma inspiré de celui qui a été mis en place à l'INSA de Lyon $^{77}$. Dans ce schéma, on peut observer l'articulation entre la plate-forme Moodle, le serveur ORI de l'INSA et celui de UNIT

- régler la question des contrats d'auteur : les points clés du contrat d'auteur sont proposés en annexe selon le modèle élaboré par l'Université de Rennes $1^{78}$

- $\quad$ gérer les contacts avec les UNT pour l'échange de métadonnées

- $\quad$ étudier la faisabilité d'une recherche fédérée catalogue du SCD et base OAI des ressources pédagogiques

La question de la mise en production d'ORI-OAI est délicate car l'installation et le paramétrage sont réputés complexes ${ }^{79}$. Cependant, l'Université de Limoges dispose déjà de compétences en interne soit auprès de l'IGE chargé du paramétrage de la base IUT en ligne, soit auprès d'un personnel du SCD dont les missions comprennent à 10\% ORI-OAI. Le recrutement d'un IGE contractuel comme programmé à l'Université de Poitiers ${ }^{80}$ est une alternative plus risquée. Il semble plus judicieux de s'appuyer sur des compétences en interne, voire de les développer et de solliciter un partenariat avec l'Université de Poitiers qui s'engage sur un projet similaire.

Dans la mesure où ORI-OAI est un logiciel libre, c'est un projet qui a l'avantage de pouvoir se mettre en place à un coût direct relativement faible (un serveur ou plusieurs serveurs sont néanmoins nécessaires) mais qui demandera beaucoup de temps et de compétences techniques à développer en interne. Ceci représente un coût important en termes de ressources humaines.

\footnotetext{
${ }^{76}$ Système d'Information Global

${ }^{77}$ Voir en annexe 1 page 50

${ }^{78}$ Voir en annexe 2 page 51

${ }^{79}$ Voir le schéma d'architecture ORI-OAI en annexe 3 page 52

${ }^{80}$ Voir profil de poste en annexe 4 page 53
} 
Le SCD doit aussi se positionner en tant que spécialiste de la description des ressources pédagogiques dans le format SupLOMFR ${ }^{81}$. Cela suppose donc que des compétences soient acquises au travers d'un stage de formation continue : compter 3 jours de formation à l'ENSSIB pour un stage sur l'indexation des ressources pédagogiques plus la mise au point d'un guide d'indexation en interne. Par ailleurs, il serait utile d'anticiper dès que possible en inscrivant des catalogueurs aux formations dispensées par les $\mathrm{CFCB}^{82}$ aux nouveaux formats de catalogage. ${ }^{83}$

Enfin, la participation au groupe de travail inter-UNT " indexation SupLOMFR " est une action à envisager afin de développer des compétences dans un domaine qui appartient aux bibliothécaires.

\subsubsection{Impact et évaluation}

Dans ce scénario, le SCD de Limoges participe au pilotage du projet et intègre les ressources pédagogiques comme une ressource supplémentaire dans son offre de contenus. Il en assure la visibilité au travers de son portail. Il entretient un partenariat avec les UNT en offrant le moissonnage de ses ressources pédagogiques ou en récupérant les métadonnées des UNT qui l'intéressent.

Le SCD développe de nouvelles compétences en matière d'indexation dans un format XML. Cette évolution peut amener à repenser un ou plusieurs postes de catalogueurs en les orientant vers les nouveaux formats de catalogage des documents numériques.

Il convient à terme d'évaluer le projet en mesurant le nombre de ressources indexées, la qualité de l'indexation, la visibilité de ces ressources. La qualité de la description est primordiale puisqu'elle permettra d'échanger et de fournir des notices de qualité aux UNT.

\subsection{Scénario 3 : Le SCD courtier pédagogique}

\subsubsection{Description et objectifs}

Dans ce scénario, le SCD " économise " la phase technique de référencement et d'indexation des ressources pédagogiques ou de moissonnage de métadonnées. Il met l'accent sur la formation des usagers et valorise les ressources des UNT en les prescrivant à ses utilisateurs dans une relation privilégiée que l'on qualifiera de courtage en information.

Cette idée est inspirée d'un article déjà cité précédemment ${ }^{84}$. Pierre Moeglin dans cet article de 2007 explique que l'on pourrait donner une quatrième vie aux ressources pédagogiques numériques, les trois vies actuelles étant le présentiel, l'autoformation et l'autodidaxie. Pierre Mœglin esquisse le profil du courtier éducatif, appelé à être soumis à l'épreuve du terrain. Le

\footnotetext{
${ }^{81}$ Schéma SupLOMFR en annexe 5 page 53

${ }^{82} \mathrm{CFCB}$ : Centre de Formation aux carrières des bibliothèques

${ }^{83}$ Un stage " Initiation à XML " est organisé par Médiadix en novembre 2010. Un autre stage est proposé sur les " métadonnées pour les bibliothèques numériques "

${ }^{84}$ MOEGLIN, Pierre. Le professeur et le courtier : une quatrième vie pour les ressources des campus numériques? in : Etudes de Communication, Numéro spécial, 2007/2
} 
courtier d'un campus numérique serait doublé d'un agrégateur, aussi bien des ressources que de la demande, garantissant simultanément la qualité de la formation dispensée et le niveau des compétences acquises.

Dans ce scénario, l'idée est de former une équipe " référente " au SCD pour les ressources pédagogiques : cette équipe aura des compétences mixtes non seulement documentaires mais aussi pédagogiques et techniques. En effet, il devient de plus en plus courant de faire face à des demandes qui dépassent la demande de renseignement documentaire au sens strict. Les ressources des UNT se présentent dans les formats les plus variés (audio, vidéos, médias interactifs) et demandent souvent une expertise technique en plus de l'expertise documentaire. Par ailleurs, l'utilisation des ressources pédagogiques en ligne nécessite un accompagnement pédagogique.

La valorisation des ressources pédagogiques des UNT passe donc par un plan d'information et de communication positionnant le SCD comme interlocuteur privilégié. En effet, si l'enseignant est prescripteur des ressources qu'il a créées et déposées sur la plate-forme Moodle, il est plus rarement prescripteur des ressources créées par d'autres enseignants par méfiance ou méconnaissance.

\subsubsection{Mise en oeuvre au SCD de Limoges}

Le projet peut s'articuler en trois phases :

\subsubsection{Formation des personnels}

- former tous les personnels des sections du SCD aux UNT : une présentation dans chaque section est nécessaire pour sensibiliser les personnels. Il faut encourager chacun à s'approprier ces nouvelles ressources en s'inscrivant dans les cours des UNT, en exploitant les cours de méthodologie et en préparant le C2i grâce aux ressources des UNT

- renforcer les compétences techniques des personnels dans chaque section afin de répondre à des demandes techniques audio ou vidéo liées à l'utilisation des ressources des UNT

- renforcer les compétences pédagogiques des personnels en investissant sur des formations de formateurs. Des formations de formateurs sont régulièrement proposées par le centre régional de formation aux carrières des bibliothèques ${ }^{85}$, mais il serait aussi judicieux de sortir du SCD et de s'inscrire dans les volets de formation des universités qui proposent également ces formations. Ces formations seraient une occasion de rencontre avec d'autres personnels de l'université ayant des fonctions de formateurs.

${ }^{85} \mathrm{http}: / /$ mco.univ-poitiers.fr/ 


\subsubsection{Plan de communication}

- $\quad$ s'entourer du matériel d'information et de description des ressources des UNT en préparant des fiches descriptives de chaque UNT et en sollicitant les UNT qui proposent des affiches et des dépliants

- disposer d'un espace sur la plate-forme Moodle pour y déposer des cours de méthodologie et des fiches techniques sur les UNT

- $\quad$ s'insérer dans les réseaux sociaux mis en place par les UNT : forums ou autres outils. La présence sur ces réseaux offre des opportunités de dialogue et de services auprès des étudiants

- $\quad$ envisager l'intégration du widget " Ubib "86 sur les sites des UNT comme cela a été fait sur la plate-forme Ebsco ou AtoZ. Ce service qui fonctionne en mode synchrone et en mode asynchrone, permet de répondre aux questions des usagers qui accèdent de plus en plus à la documentation à distance et dans des environnements de type web 2.0

- réfléchir à la mise en valeur des ressources des UNT sur le site web du SCD

\subsubsection{Plan de formation}

- $\quad$ intégrer dans les plans de formation du SCD une présentation des ressources des UNT visant prioritairement les L1 dès le premier semestre. Les ressources des UNT s'adressent en effet très souvent aux premières années et présentent l'avantage d'être accessibles facilement

- $\quad$ engager un travail d'interaction avec l'étudiant en proposant systématiquement les cours UNT en complément des manuels dont le nombre d'exemplaires est parfois insuffisant, en expliquant comment l'UNT peut être un complément au moment des examens

- $\quad$ apprendre à utiliser la fonction cartable de IUT en ligne ou celle de Canal-U vidéo pour prescrire des ressources adaptées aux demandes récurrentes des étudiants

- $\quad$ s'insérer dans les dispositifs de formation au $\mathrm{C} 2 \mathrm{i}$ là où les compétences des bibliothécaires peuvent s'exercer, en particulier dans les modules de recherche d'information ${ }^{87}$

\subsubsection{Impact et évaluation}

L'impact de ce scénario peut être assez fort puisqu'il renforce la mission formation du SCD. Le courtage éducatif contribuerait à renforcer l'utilité et la viabilité des UNT.

Au delà du simple rôle de prescription des ressources, les bibliothécaires peuvent jouer un rôle plus actif. En plus d'identifier, repérer, signaler et veiller à la qualité des ressources, les bibliothécaires peuvent assurer d'autres fonctions dont certaines seront d'ordre technique telles que s'assurer de la disponibilité des bases, veiller à la mise à jour des logiciels permettant

\footnotetext{
${ }^{86}$ Ubib : service de référence à distance mis en place par les universités du réseau Ouest Atlantique (RUOA). Le SCD de Limoges adhère à Ubib depuis début 2010. Site web : http://www.ubib.fr/

${ }^{87}$ Module B2 du C2i intitulé " Rechercher l'information " qui comprend : distinguer les différents types d'outils de recherche, formaliser les requêtes de recherche et récupérer et savoir utiliser les informations (texte, image, son, fichier, pilote, application,...)
} 
d'accéder aux ressources, disposer des outils permettant le bon fonctionnement des produits alors que d'autres seront d'ordre pédagogique. De nouvelles formes d'apprentissage apparaissent avec les podcasts ou les serious games qui deviennent des outils d'apprentissage au même titre que les manuels. Mais l'apprentissage nécessite aussi des contacts sociaux que les étudiants peuvent trouver dans les bibliothèques universitaires.

Le renforcement de cette mission de formation pourrait être l'un des moyens de contribuer au plan de soutien des étudiants décrocheurs mis en place à l'Université de Limoges ${ }^{88}$.

En terme d'évaluation, il conviendrait de se fixer des indicateurs en nombre d'étudiants formés, nombre d'heures de formation, nombre de personnels impliqués.

\subsection{BiLAN}

Ces trois scénarios proposent des approches diamétralement opposées. Faut-il se préoccuper de fournir une indexation de qualité en amont pour assurer la meilleure visibilité possible, ou est-il préférable de se placer au plus près des utilisateurs en privilégiant la démarche de formation à l'utilisation des ressources? Au regard de la relative sous-utilisation des ressources des UNT, le troisième scénario est celui qui aurait la préférence sur les deux premiers.

Cependant, un travail d'indexation de qualité aurait l'avantage de pérenniser les ressources en les rendant aisément accessibles à des usagers de plus en plus dispersés ou éloignés physiquement des campus. De plus, le deuxième scénario avec la mise en place d'ORI-OAI ouvre la voie à l'indexation et au référencement d'autres ressources électroniques : archives ouvertes, thèses, revues en ligne...

Chacun de ces scénarios fait appel à des compétences maîtrisées par les bibliothécaires tout en ouvrant le champ vers l'acquisition de nouvelles compétences : compétences techniques dans l'hypothèse des deux premiers scénarios, compétences pédagogiques dans le troisième scénario.

${ }^{88}$ UNIVERSITE DE LIMOGES. Les étudiants décrocheurs [en ligne]. 2009. Disponible sur :

$<$ http://www.carrefourdesetudiants.unilim.fr/IMG/pdf/DecrocheursV7.pdf > (consulté le 5 novembre 2010) 
En soulevant la question des ressources pédagogiques en ligne, et en particulier les ressources des Universités Numériques Thématiques, Claudie Madjarev a soulevé très justement la question de notre rôle dans l'Université des années à venir. Nous savons que nous devons être attentif à notre environnement, à l'évolution des pratiques et des usages, à l'émergence de nouvelles ressources.

Or, cette question semble peu débattue au sein des bibliothèques universitaires. En témoignent le peu d'articles disponibles dans le Bulletin des Bibliothèques de France, sur les blogs professionnels ou le nombre relativement restreint de bibliothécaires rencontrés lors des Journées Vivaldi à Limoges ou du CIUEN 2010.

Le SCD de Limoges a su s'adapter à l'évolution de la documentation numérique et les nouveaux usages induits. Son rôle pilote pour les thèses électroniques, la numérisation des herbiers ou son rôle d'éditeur des cahiers de sémiotique l'ont démontré. L'apparition et le développement des ressources pédagogiques en ligne dans l'offre de contenus constituent une nouvelle opportunité à saisir. 



\section{Bibliographie}

\section{Ressources PÉdAgogiQUes, INDEXATION et RÉFÉRENCEMENT}

[1] AGENCE BIBLIOGRAPHIQUE DE L'ENSEIGNEMENT SUPERIEUR.

Expérimentation WebDewey [en ligne]. 2010. Disponible sur : <

http://fil.abes.fr/2010/02/19/experimentation-webdewey/ > (consulté le 22 février 2010)

[2] ASSOCIATION FRANÇAISE DE NORMALISATION (AFNOR). Technologies de l'information pour l'éducation, la formation et l'apprentissage : profil français d'application du LOM (LOMFR) : Métadonnées pour l'enseignement. NF Z 76-040. 2006

[3] BERTIN, Gilles, BERTRAND, Annie, BOURDA, Yolaine, CHARTRON, Ghislaine, CHERHAL, Elisabeth, FLORY, Laurent, GÓMEZ DE REGIL, Rosa Maria, HENNEQUIN, Xavier, JOLY, Monique, MARINO, Jean-Bernard, PERNIN, Jean-Philippe. L'indexation des ressources pédagogiques numériques : un partenariat à créer entre les $S C D$ et les services TICE au sein des universités [en ligne]. 2004. Disponible sur : < http://www.enssib.fr/bibliotheque-numerique/notice-1232 > (consulté le 31 janvier 2010)

[4] BOURDENET, Philippe, WOLFARTH, Jennifer. Indexation : une collaboration SCD -TICE in : Arabesques n 56, octobre, novembre, décembre 2009. p. 15-16

[5] BOURGES, Raymond, COLMANT, Yohan. HUET, Nolwen, JANNIN, François. L'architecture ORI-OAI in : LEBLOND, Corinne, Dir. Archivage et stockage pérennes : enjeux et réalisations. Paris : Lavoisier, 2009, 224 p. (Traité des sciences et techniques de l'information)

[6] CENTRE REGIONAL DE DOCUMENTATION PEDAGOGIQUE (Bordeaux). Les métadonnées [en ligne]. 2005. Disponible sur : < http://crdp.acbordeaux.fr/documentalistes/formation/metadonnees.asp > (consulté le 25 février 2010)

[7] DA SILVA, Christophe. Vers une collaboration entre les services producteurs de ressources pédagogiques et les SCD : état des lieux et perspectives. 2007. Mémoire DCB. ENSSIB

[8] FICHEZ, Elisabeth. Acteurs des TICE et contexte de l'enseignement supérieur : TICE et tendances pédagogiques au long d'innovations annoncées [en ligne]. 2008. Disponible sur : $<$ http://w3.u-grenoble3.fr/les_enjeux/2008-supplement/Fichez/index.php > (consulté le 20 juillet 2010)

[9] FRANCE. MINISTÈRE DE L'ENSEIGNEMENT SUPÉRIEUR ET DE LA RECHERCHE; FRANCE. MINISTÈRE DE L'EDUCATION NATIONALE. Indexation et 
visibilité des ressources pédagogiques [en ligne]. 2007. Disponible sur : <

http://www.educnet.education.fr/dossier/metadata/seminaire-sdtice-2007 > (consulté le 30 janvier 2010)

[10] FRANCE. MINISTÈRE DE L'EDUCATION NATIONALE; FRANCE. MINISTÈRE DE L'ÉDUCATION NATIONALE. Indexation et visibilité des ressources pédagogiques : un enjeu pour la valorisation du patrimoine pédagogique des établissements d'enseignement supérieur [en ligne]. 2007. Disponible sur : <

http://www.educnet.education.fr/chrgt/seminaire_indexation_2007.pdf $>$ (consulté le 20 juillet 2010)

[11] MERMET, Jean-Michel, CARRERE, Christine. ARPEM : une expérience concrète de mutualisation sur le campus grenoblois in : Document numérique, $\mathrm{n}^{\circ} 1 / 2003$, Vol. 7, p. 141156

[12] MOEGLIN, Pierre. Le professeur et le courtier : une quatrième vie pour les ressources des campus numériques? in : Etudes de Communication, Numéro spécial, 2007/2

[13] MONTEIL, Alain. Archivage de Ressources Pédagogiques Multimédias (ARPEM). Suivi du développement et mise en place des procédures humaines [en ligne]. 2002. Disponible sur : < http://www.enssib.fr/bibliotheque/documents/dessid/rsmonteil.pdf > (consulté le 5 décembre 2010)

[14] REBAT, Marie-Thérèse. Rôle nouveau des SCD dans le domaine de la valorisation des ressources pédagogiques en ligne [en ligne]. 2003. Disponible sur : $<$ http://mediat.upmfgrenoble.fr/61392489/0/fiche_pagelibre/\&RH=MEDIATFR_PRES\&RF=MEDIATFR_PR OF $>$ (consulté le 31 janvier 2010)

\section{UNIVERSITÉS NUMÉRIQUES}

[1] BARTHE, Emmanuel. Les missions de l'Université numérique juridique francophone (UNJF) [en ligne]. 2009. Disponible sur : < http://www.precisement.org/blog/Les-missionsde-l-Universite.html > (consulté le 30 janvier 2010)

[2] CIUEN. L'Université à l'Ère du numérique : du 14 au 16 juin 2010 à Strasbourg [en ligne]. 2010. Disponible sur : < http://www.ciuen.fr/ > (consulté le 31 Janvier 2010)

[3] DANQUIGNY, Thierry. Réseau social Unisciel [en ligne]. 2009. Disponible sur : < http://www.knowtex.com/tag/UNT > (consulté le 14 juin 2010)

[4] Entretien avec le Professeur Albert-Claude Benhamou, promoteur des UNT in : Distances et savoirs, Vol. 4, n²006/1, p. 99-107

[5] FICHEZ, Elisabeth. Campus numériques français : pertinence des notions de réussite et d'échec in : Etudes de Communication, Numéro spécial, 2007/2 
[6] FRANCE. MINISTÈRE DE L'ENSEIGNEMENT SUPÉRIEUR ET DE LA

RECHERCHE. Faire du numérique un outil pédagogique et au service de la vie étudiante [en ligne]. 2009. Disponible sur : < http://www.enseignementsup-

recherche.gouv.fr/cid22726/faire-du-numerique-un-outil-pedagogique-et-au-service-de-la-vieetudiante.html $>$ (consulté le 14 juin 2010)

[7] FRANCE. MINISTÈRE DE L'ENSEIGNEMENT SUPÉRIEUR ET DE LA

RECHERCHE. Universités numériques [en ligne]. 2009. Disponible sur : < http://www.universites-numeriques.fr/fr > (consulté le 30 Janvier 2010)

[8] FRANCE.MINISTÈRE DE L'ENSEIGNEMENT SUPÉRIEUR ET DE LA RECHERCHE. 16 millions d'euros pour développer le numérique à l'Université. [en ligne]. 2009. Disponible sur : < http://www.enseignementsup-recherche.gouv.fr/cid28954/16millions-d-euros-pour-developper-le-numerique-a-l-universite.html > (consulté le 31 janvier 2010)

[9] FRANCE.MINISTÈRE DE L'ENSEIGNEMENT SUPÉRIEUR ET DE LA RECHERCHE. Enquête sur l'université numérique [en ligne]. 2009. Disponible sur : $<$ http://media.enseignementsuprecherche.gouv.fr/file/Universite_numerique/49/8/enquete_65498.pdf $>$ (consulté le 30 janvier 2010)

[10] ISAAC, Henri. L'Université numérique : rapport à Madame Valérie Pécresse, Ministre de l'enseignement supérieur et de la recherche [en ligne]. 2008. Disponible sur : $<$ http://media.enseignementsuprecherche.gouv.fr/file/Nouvelle_universite/55/7/universitenumerique_23557.pdf $>$ (consulté le 1er septembre 2010)

[11] L'ETUDIANT. Les universités numériques thématiques en fiches [en ligne]. 2009. Disponible sur : < http://www.educpros.fr/dossiers/les-universites-numeriques-thematiquesen-fiches/h/0b68e6ea6c/d/984/a/unjf-universite-numerique-juridique-francophone.html > (consulté le 31 janvier 2010)

[12] MINISTÈRE DE LA JUSTICE ET DES LIBERTÉS. Université numérique juridique française : le droit en ligne [en ligne]. 2009. Disponible sur : <

http://www.justice.gouv.fr/index.php?rubrique $=10030 \&$ article $=18127>$ (consulté le 30 janvier 2010)

[13] PAQUELIN, Didier. Dir. CIUEN 2008 : actes du colloque international "L'université à l'ère du numérique ", Bordeaux, 10-12 décembre 2008

[14] THIBAULT, Françoise. Autour des campus numériques français : Repères dans les initiatives du ministère en charge de l'enseignement supérieur in : Distances et Savoirs, 2006/1, Vol. 4, p. 109-112

[15] UNISCIEL. Appel à projets 2010 [en ligne]. 2010. Disponible sur : < http://www.unisciel.fr/pg/file/beaugeois/read/3674/volet-2a-recensement-des-ressourcesexistantes-dans-votre-\%EF\%BF\%BDtablissement-destin $\%$ EF\%BF $\%$ BDes- $\%$ EF\%BF\%BD$\%$ EF\%BF\%BDtre-mutualis\%EF\%BF\%BDes-au-sein-d\%EF\%BF\%BDunisciel > 
[16] UNIVERSITE DE LYON. Un exemple de mutualisation, l'UNJF par Yann Tanguy [en ligne]. 2009. Disponible sur $:<$ http://suel.univ-lyon3.fr/eltv?task=viewvideo\&video_id=335 $>$ (consulté le 30 janvier 2010)

\section{UnIVERSITÉ DE Limoges}

[1] AGENCE D'EVALUATION DE LA RECHERCHE ET DE L'ENSEIGNEMENT SUPERIEUR (AERES). Rapport d'évaluation de l'Université de Limoges [en ligne]. 2008. Disponible sur : $<$ http://www.aeresevaluation.fr/content/download/12412/177581/file/AERES-S1-Limoges.pdf> (consulté le 27 août 2010)

[2] UNIVERSITE DE LIMOGES. Les étudiants décrocheurs. [en ligne]. 2009. Disponible sur : < http://www.carrefourdesetudiants.unilim.fr/IMG/pdf/DecrocheursV7.pdf > (consulté le 5 novembre 2010)

[3] UNIVERSITE DE LIMOGES. L'université de Limoges ... un peu d'Histoire [en ligne]. 2010. Disponible sur : < http://www.unilim.fr/spip.php?article658\&lang=fr $>$ (consulté le 20 juillet 2010)

[4] UNIVERSITE DE LIMOGES. Tableau de bord de l'Université : TABUL [en ligne]. Disponible sur : <http://www.unilim.fr/spip.php?article642\&lang=fr $>$ (Consulté le 14 juin 2010)

\section{Sites Web}

[1] LOM-fr [en ligne]. 2010. Disponible sur : < http://www.lom-fr.fr/ > (consulté le 30 janvier 2010)

[2] ORI-OAI [en ligne]. 2010. Disponible sur : < http://www.orioai.org/display/ORIOAI/ORI-OAI.ORG > (consulté le 30 janvier 2010)

[3] SupLOMFR [en ligne]. 2010. Disponible sur : <http://www.sup.lomfr.fr/index.php? title $=$ Accueil $>$ (consulté le 30 janvier 2010)

[4] Universités Numériques [en ligne]. 2010. Disponible sur : $<$ http://www.universitesnumeriques.fr/fr> (consulté le 30 août 2010) 
ANNEXE 1 : SCHÉMA GÉNÉRAL DU WORKFLOW À L'INSA DE LYON..................50 ANNEXE 2 : LES POINTS CLÉS DU CONTRAT D'AUTEUR........................................51

ANNEXE 3 : LES MODULES DU LOGICIEL ORI-OAI...................................................52

ANNEXE 4 : FICHE DE POSTE ITRF......................................................................53

ANNEXE 5 : CARTE HEURISTIQUE SUPLOMFR..........................................................54 


\section{Annexe 1 : Schéma général du workflow à I'INSA de Lyon}

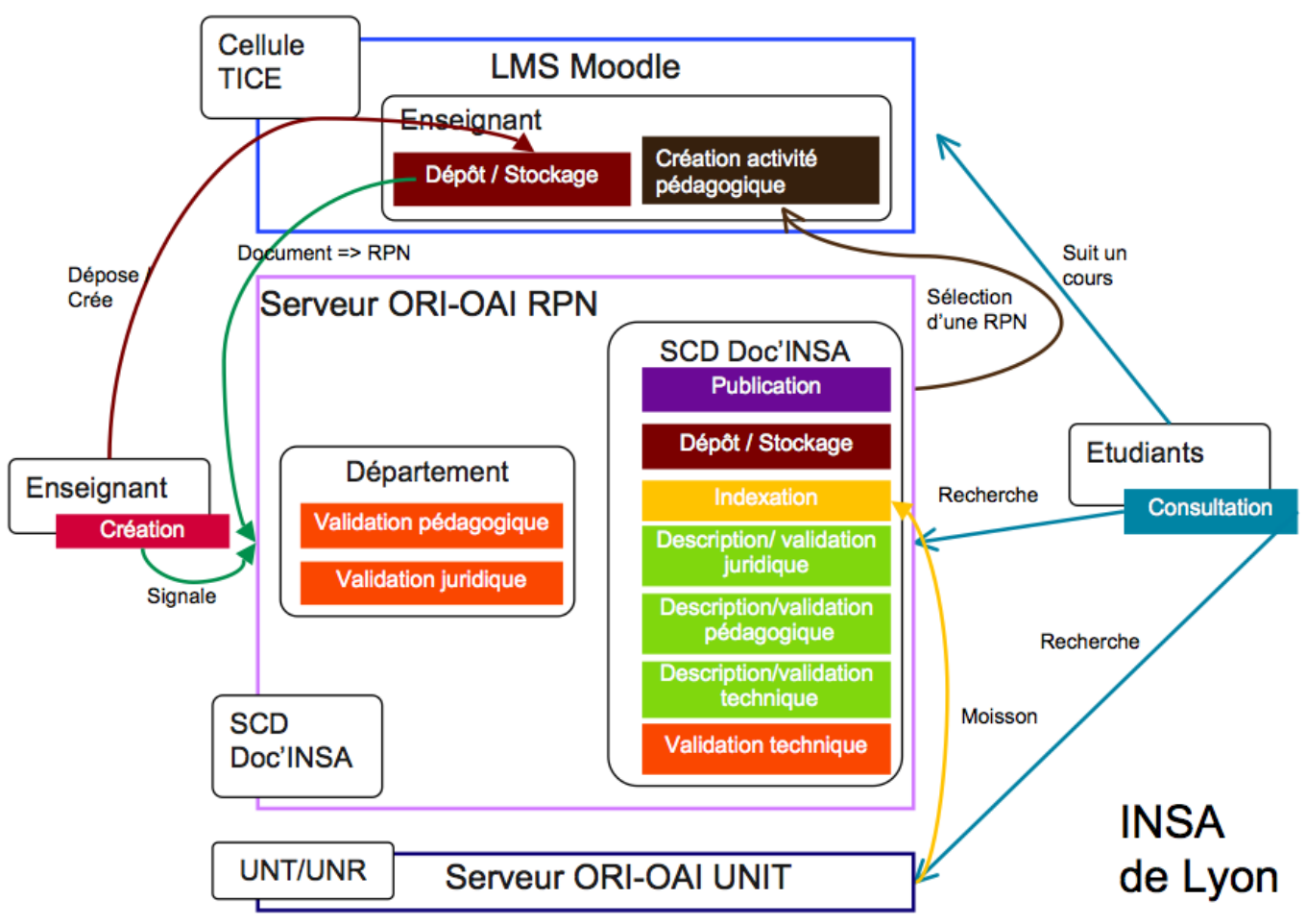

Source : INSA de Lyon. Document diffusé lors du stage ENSSIB 2010 sur l'indexation des ressources pédagogiques. Stage sous la responsabilité pédagogique de Mme Monique Joly 


\section{Annexe 2 : les points clés du contrat d'auteur}

\section{LES DROITS CÉDÉS}

- Cession à titre gracieux

- $\quad$ Limitée à une diffusion électronique uniquement

- $\quad$ Pas d'exploitation commerciale possible de la part de l'établissement

\section{LES GARANTIES ACCORDÉES AUX AUTEURS}

- Les moyens techniques d'exercer un contrôle direct sur la diffusion de l'œuvre

- Un accompagnement de l'établissement pour l'utilisation de ces moyens techniques, le cas échéant

- La conservation des possibilités d'exploitation distincte de l'œuvre

- L'accompagnement de l'établissement dans le choix et l'apposition de licences ou mentions légales sur leur œuvre

- La possibilité d'être accompagné et/ou assisté par l'établissement dans leurs démarches en cas d'atteinte de leur droit d'auteur

- L'accompagnement de leur établissement dans les formalités d'acquisition de droits sur des œuvres protégées

(C) 2010 Caroline Gravier, Centre d'Ingénierie et de Ressources Multimédia (CIRM), Université de Rennes 1 


\section{Annexe 3 : les modules du logiciel ORI-OAI}

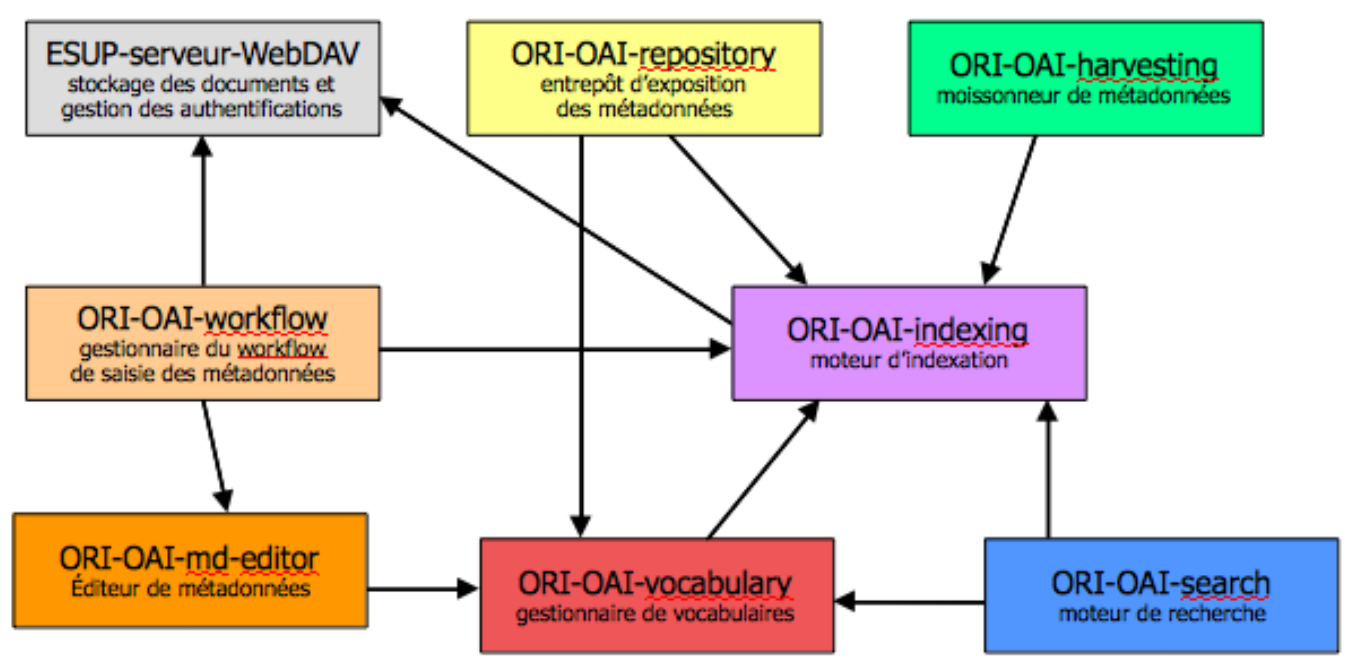

- 8 modules

- interopérables

Source : site internet ORI-OAI www.ori-oai.org/ 


\section{Annexe 4 : fiche de poste ITRF}

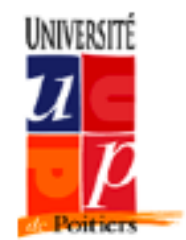

FICHE de POSTE ITRF

ANNEE 2010

Etablissement : Université de Poitiers

Composante : I-médias

Localisation du poste : I-médias campus et SCD BU Droit-Lettres

Adresse de la composante d'affectation :
POSTE ITRF Catégorie
A $\otimes \quad$ B
C

CORPS : IGE

BAP : F

Emploi type : Chargé de système d'information documentaire Code : F2A22

区 Concours externe (CE)

Missions de l'agent :

\section{DESCRIPTION DU POSTE}

- Recenser les ressources numériques produites par l'Université de Poitiers et les projets en la matière: ressources pédagogiques, édition électronique, thèses électroniques, numérisation, archives ouvertes, production vidéo

- Proposer et mettre en cuvre un schéma général de gestion, indexation, signalement, diffusion et valorisation.

Activités essentielles :

- Définir tout ou partie des fonctionnalités d'un nouveau système d'information en cohérence avec le référentiel de l'établissement

- Assurer l'administration utilisateurs de plateformes de dépôt et d'archivage institutionnel (validation des métadonnées de gestion, gestion des habilitations, ...)

- Assurer le référencement et l'accessibilité des textes intégraux des documents produits par l'établissement sous forme électronique

- Assurer une assistance à l'utilisation de ces plateformes

- Gérer les outils de diffusion de l'information évaluer et suivre leur évolution

- Mettre en place des indicateurs d'évaluation et d'usage du nouveau système d'information documentaire

- Organiser la formation des personnels aux nouveaux outils et nouvelles procédures, accompagner l'évolution des pratiques professionnelles

- Appliquer une démarche qualité

- Assurer une veille technologique sur les méthodes et outils adaptés aux besoins de l'information scientifique et technique : technologies émergentes, suivi d'évolution de produits (plateformes éditoriales, outils d'application)

Source : Site internet de l'Université de Poitiers http://univ-poitiers.fr 


\section{Annexe 5 : Carte heuristique SupLOMFR}

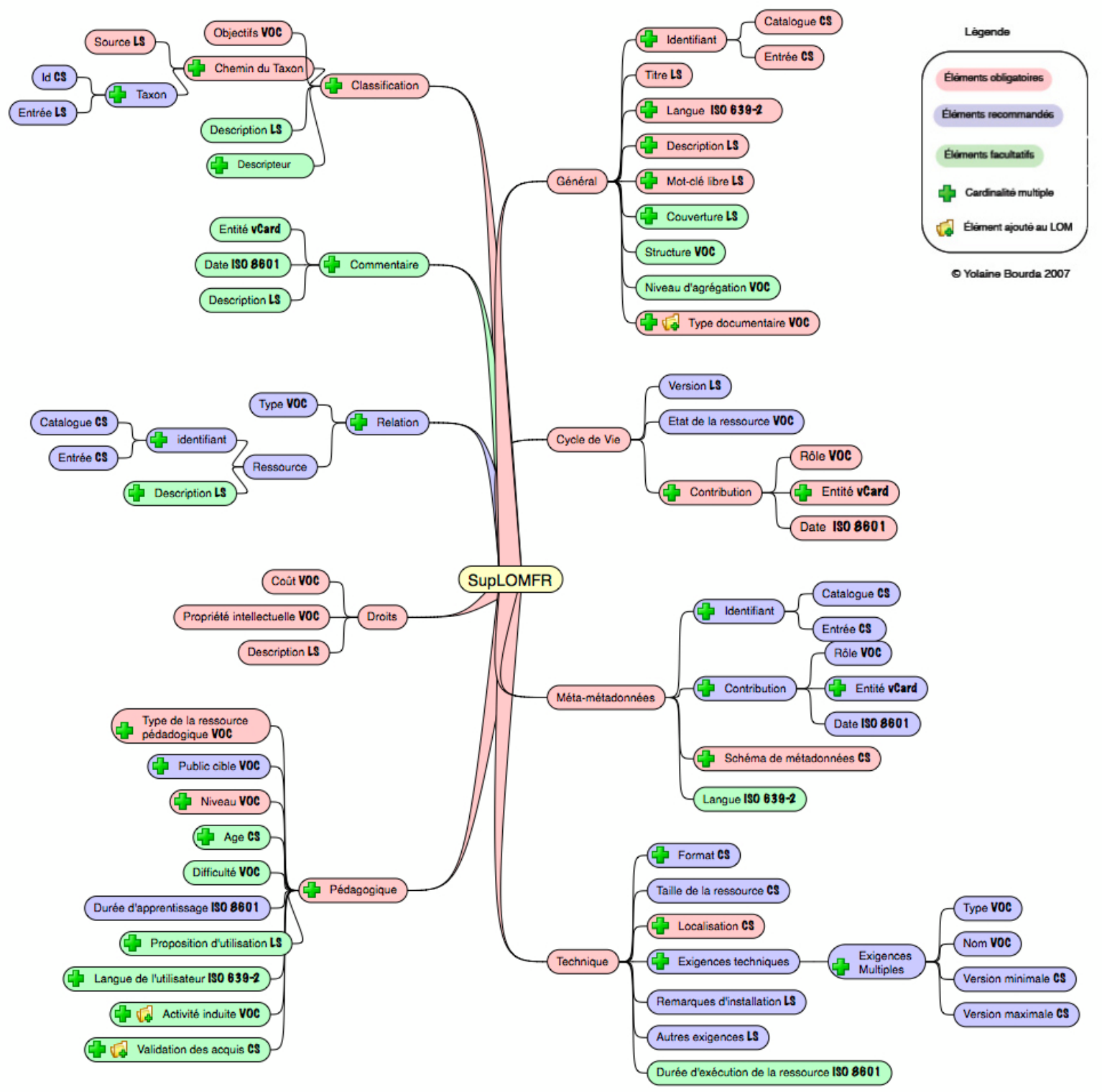

Source : site internet SupLOMFR http://www.sup.lomfr.fr/index.php?title=Accueil 